\section{ENGEVISTA}

Página da revista: http://www.uff.br/engevista/seer/

\title{
Parallel moving mechanical systems kinematics
}

\author{
Florian Ion Petrescu ${ }^{1}$ \\ Relly Victoria Petrescu ${ }^{2}$
}

\begin{abstract}
Moving mechanical systems parallel structures are solid, fast, and accurate. Between parallel systems it is to be noticed Stewart platforms, as the oldest systems, fast, solid and precise. The work outlines some few main elements of Stewart platforms. It begins with the geometry platform and structure, and then one presents the kinematic elements of it, positions, velocities and accelerations. If a structural motto element consists of two moving elements which translate relative, drive train and especially dynamic it is more convenient to represent the motto element as a single moving component. One has thus seven moving parts (the six motto elements or feet to which is added mobile platform 7) and one fixed. The whole work is based on knowledge of analytic geometry, so we can consider this work as a study of the applied mathematics.
\end{abstract}

Keywords: Moving mechanical systems, Parallel structures, Stewart platforms, Inverse kinematics, Kinematic elements, Motto element, Mobile platform.

\footnotetext{
${ }^{1}$ Bucharest Polytechnic University

2 Bucharest Polytechnic University
} 


\section{Introduction}

Moving mechanical structures are used increasingly in almost all vital sectors of humanity (CAO et al., 2013). The robots are able to process integrated circuits sizes micro and nano, on which the man they can be seen even with electron microscopy (GARCIA et al., 2007). Dyeing parts in toxic environments (TONG; GU; XIE, 2013), working in chemical and radioactive environments, or at depths and pressures at the bottom of huge oceans, or even cosmic space conquest and visiting exo-planets, are now possible, and were turned into from the dream in reality, because mechanical platforms sequential gearbox (PERUMAAL; JAWAHAR, 2013).

Robots were developed and diversified, different aspects, but today, they start to be directed on two major categories: systems serial and parallel systems (PADULA; PERDEREAU, 2013). Parallel systems are more solid, but more difficult to designed and handled, which serial systems were those which have developed the most. In medical operations or radioactive environments is preferred mobile systems parallel to their high accuracy positioning (REDDY; SHIHABUDHEEN; JACOB, 2012).

\section{The structure and geometry of steward system}

Figure 1 shows the kinematic diagram of a mobile mechanical parallel system, heving all 12 kinematic couplings (which connect the six motor legs with the two platforms fixed and mobile) type ball joints (couplers sphere to sphere allowing all rotations possible and do not give permission to produce any translation), practically couplers third grade (C3). Kinematic engines couplings (six in number) may be constructed in two versions: $\mathrm{C} 5$ or $\mathrm{C} 4$.

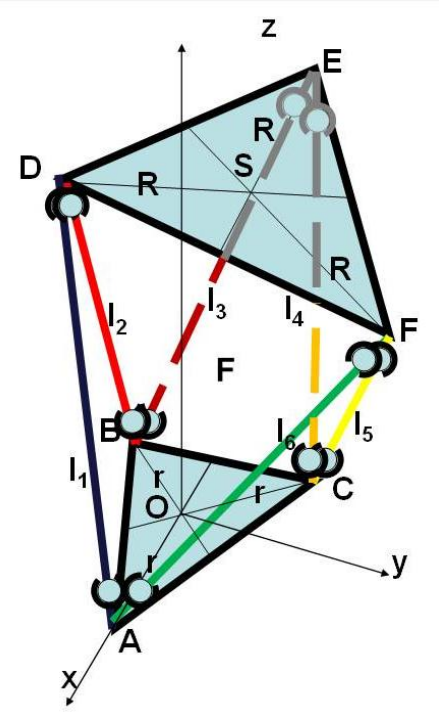

Fig. 1 Geometry of a Stewart platform 
Couplings sphere in sphere (ball joints) allow rotations in space on all three axes and stop all translations. They are more difficult to achieve in terms of technology, are more expensive and generally have shorter life, their wear being quite fast (even if the contact surface of the sphere is large sphere type). But have the great advantage of a reduced size (mass and low volume) (see Figure 2). Their life can be extended by optimal design, by careful processing, through a suitable lubricant, or using special materials.

Ball joints are used in the car industry, especially in the automotive industry.

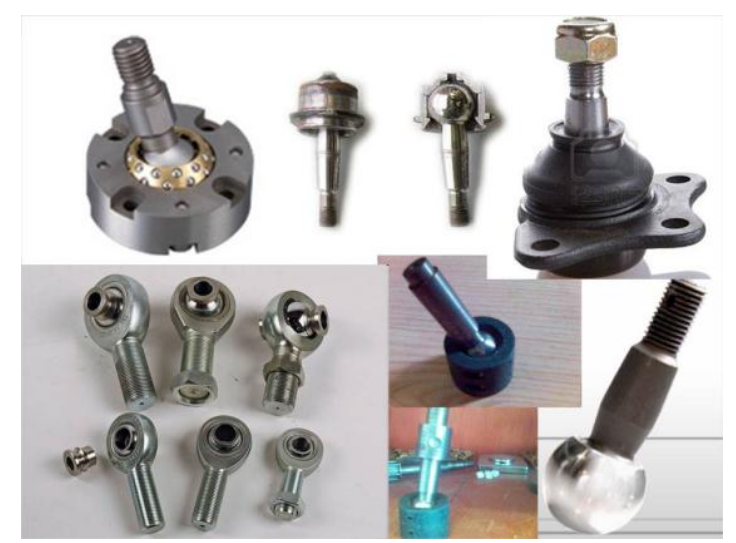

Fig. 2 Ball joints have multiple uses

For a parallel system 12 ball joint (C3), and 6 motor couplings (C5) only the translation the grade fifth, the mobility of the system (spatial mechanism) is given by the general formula (1), (for a spatial mechanism family 0 ):

$$
\begin{aligned}
& M_{0}= \\
& =6 \cdot m-5 \cdot C_{5}-4 \cdot C_{4}-3 \cdot C_{3}-2 \cdot C_{2}-1 \cdot C_{1} \\
& =6 \cdot m-5 \cdot C_{5}-3 \cdot C_{3}=6 \cdot 13-5 \cdot 6-3 \cdot 12= \\
& =78-30-36=12
\end{aligned}
$$

Where $\mathrm{m}$ represents the number of the moving parts of the mechanism (system), in this case where $\mathrm{m}$ is equal to 13 , because the six legs each consisting of two elements (ie $6 * 2=12$ ), and one of the two platforms (the upper) is also mobile (representing the thirteenth movable element of the system).

Of the 12 degrees of freedom of the system only 6 are active (they representing the linear movements of the linear motors).

The other six degrees of freedom are passive (does not indicate the need to use additional actuators to achieve them). They are basically evidenced by six additional rotational movements of the six legs, each leg consists of two elements cinematic considered as a solid, may rotate freely between its two ball joints (by which is connected to the two platforms: based on the 
fixed and movable above), (to follow Figure 3). These additional rotational movements allow the kinematic movements of the system.

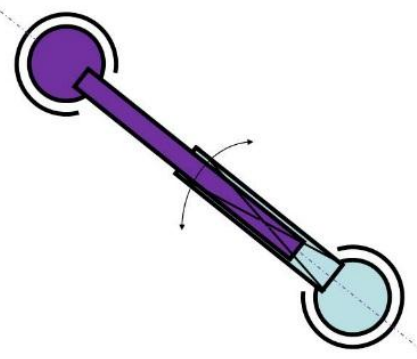

Fig. 3 Additional rotational movement of a leg between the two ball joints

Basically, it is used instead of couplers translation engines (C5) some motor couplers type cylindrical (C4) which in addition to translational movement, allow and a relative rotation between the two bars of the motor coupling. Linear actuators are constructed so that each allows a relative rotation between its two active bars. Motor movement is the linear, but is allowed and a relative rotation in motto element.

In this situation will disappear the six couplers of grade V (C5), being replaced entirely with swivels cylindrical grade IV (4), (see Figure 4). The formula of degree of mobility takes the appearance (2).

$$
\begin{aligned}
& M_{0}= \\
& =6 \cdot m-5 \cdot C_{5}-4 \cdot C_{4}-3 \cdot C_{3}-2 \cdot C_{2}-1 \cdot C_{1}= \\
& =6 \cdot m-4 \cdot C_{4}-3 \cdot C_{3}=6 \cdot 13-4 \cdot 6-3 \cdot 12= \\
& =78-24-36=18
\end{aligned}
$$

Mechanism enhances its mobility, but only six of these degree of mobility are active (they refer to linear movements imposed by the six actuators). In this case the mechanism has 12 passive rotational movements (12 passive degree of mobility).

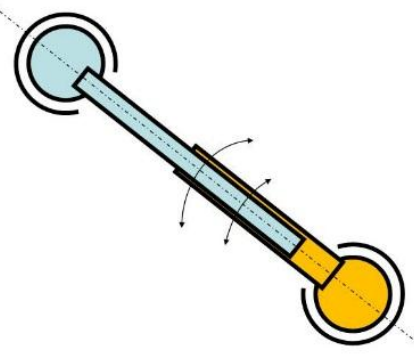

Fig. 4 Two additional rotational movements of a leg: first one is the leg rotation between the two ball joints; and the second one is the relative rotation between the two elements (cylindrical bars) of the leg 
Both variants presented are functional and have a high dynamic.

Now is frequently used a combined system more rigid (in terms of dynamic) and economic, in which six of the ball joints (C3) are replaced with six universal joints, that are couplings of the Class IV (C4). So of the 12 ball joints C3 remain now for the new system to use half (six ball joint, couplers C3), and the other six will be now of the class IV (type universal joints) and together with the old cylindrical joints with motors (C4) will achieve now to the Stewart platform 12 couplers type $\mathrm{C} 4$. Mobility will be given now by formula (3).

$$
\begin{aligned}
& M_{0}=6 \cdot m-5 \cdot C_{5}-4 \cdot C_{4}-3 \cdot C_{3}-2 \cdot C_{2}-1 \cdot C_{1}= \\
& =6 \cdot m-4 \cdot C_{4}-3 \cdot C_{3}=6 \cdot 13-4 \cdot 12-3 \cdot 6= \\
& =78-48-18=12
\end{aligned}
$$

The vast majority of parallel platforms type Stewart has today 12 universal joints and 6 cylinder motor couplings, all of them being kinematic couplings of the grade IV (C4). Disappearing joints C3 and couplings C5 engines and remain only universal joints and couplings cylinder engines, all of the kinematic class $\mathrm{C} 4$ (Fig. 5).

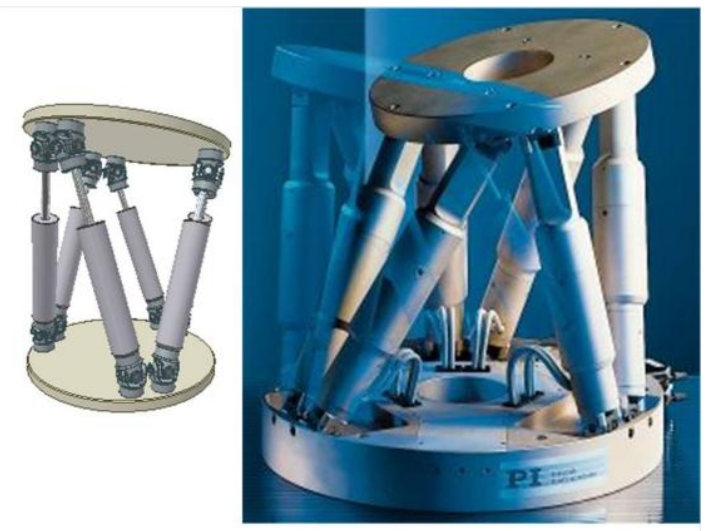

Fig. 5 Modern platform Stewart which uses only (18) couplings grade 4 (C4)

Universal joints can be used constructively in several ways (see Fig. 6).

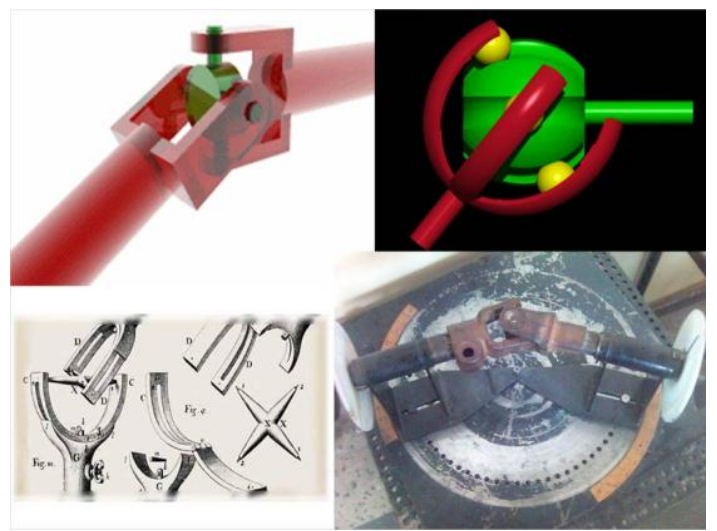

Fig. 6 Universal joints (their constructive diversity is high) 
The formula for calculating the mobility is writing now greatly simplified by expression (4).

$$
\begin{aligned}
& M_{0}= \\
& 6 \cdot m-5 \cdot C_{5}-4 \cdot C_{4}-3 \cdot C_{3}-2 \cdot C_{2}-1 \cdot C_{1}= \\
& =6 \cdot m-4 \cdot C_{4}=6 \cdot 13-4 \cdot 18= \\
& =78-72=6
\end{aligned}
$$

Today, they use various systems of type Stewart (see Fig. 7).

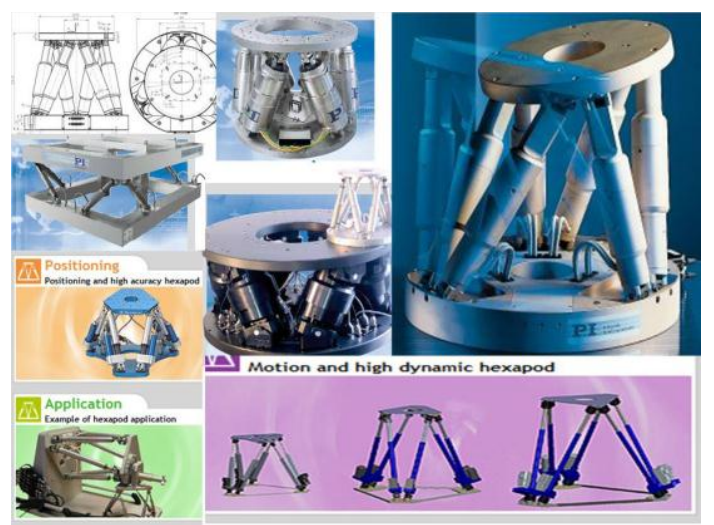

Fig. 7 Various platforms type Stewart

All these platforms achieve high speeds in work with a good dynamics. Their balancing is simpler and higher. Systems are more robust and more accurate. In Fig. 8 one can see a Stewart platform with nine legs.

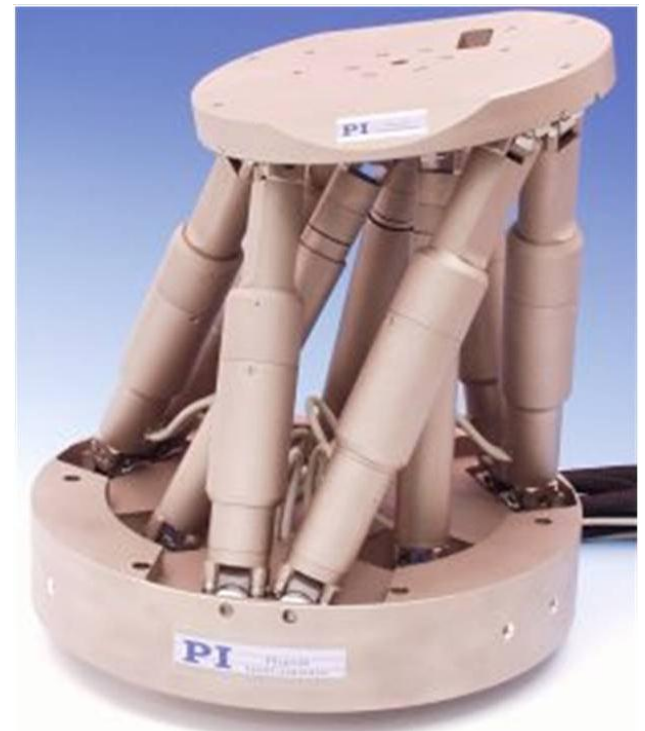

Fig. 8 A Stewart platform with nine legs Its structural formula is given by relation 5 . 


$$
\begin{aligned}
& M_{0}= \\
& 6 \cdot m-5 \cdot C_{5}-4 \cdot C_{4}-3 \cdot C_{3}-2 \cdot C_{2}-1 \cdot C_{1}= \\
& =6 \cdot m-4 \cdot C_{4}=6 \cdot 19-4 \cdot 27= \\
& =114-108=6
\end{aligned}
$$

From the structural formula can be seen that only six legs are engines and the other three are driven by the mobile platform element (their role being more to increase system stability). Or all legs are motors and their movements must be well coordinated and synchronized with other main actuators (Calculation of movement in this system is much more difficult). To have the mobility of this mechanism 9 instead of 6 as three universal joints must be replaced with three spherical kinematic couplings (see relation 6).

$$
\begin{aligned}
& M_{0}= \\
& 6 \cdot m-5 \cdot C_{5}-4 \cdot C_{4}-3 \cdot C_{3}-2 \cdot C_{2}-1 \cdot C_{1}= \\
& =6 \cdot m-4 \cdot C_{4}-3 \cdot C_{3}=6 \cdot 19-4 \cdot 24-3 \cdot 3= \\
& =114-96-9=9
\end{aligned}
$$

Stewart systems can keep their balance during rapid movements loaded with a heavy load (see Fig. 9).

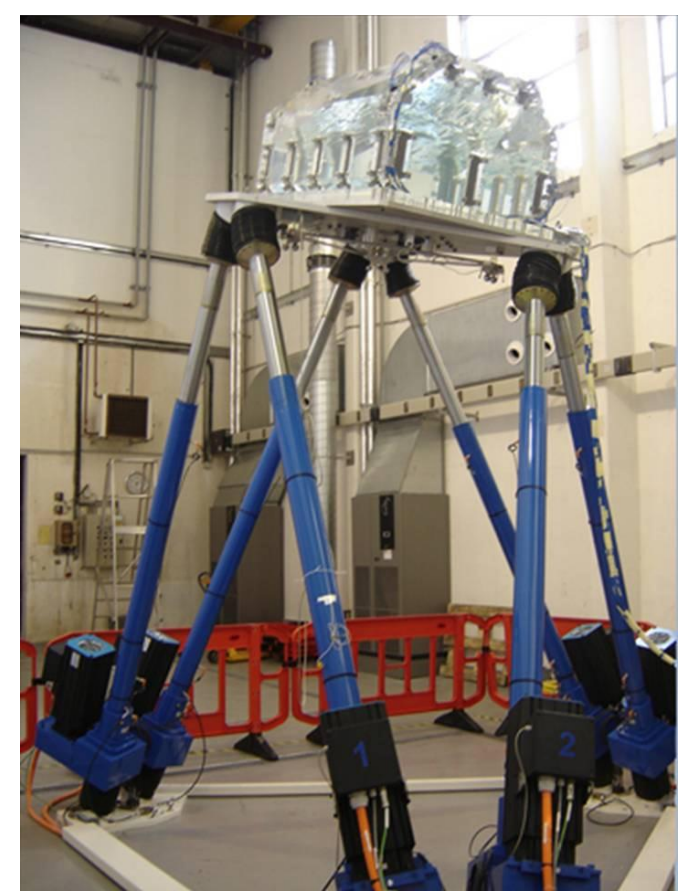

Fig. 9 A high loaded Stewart platform 
ISSN: $1415-7314$

ISSN online: 2317-6717

\section{Inverse kinematics}

\section{Determining positions and displacements}

In Fig. 10 one can see a simple theoretical model.

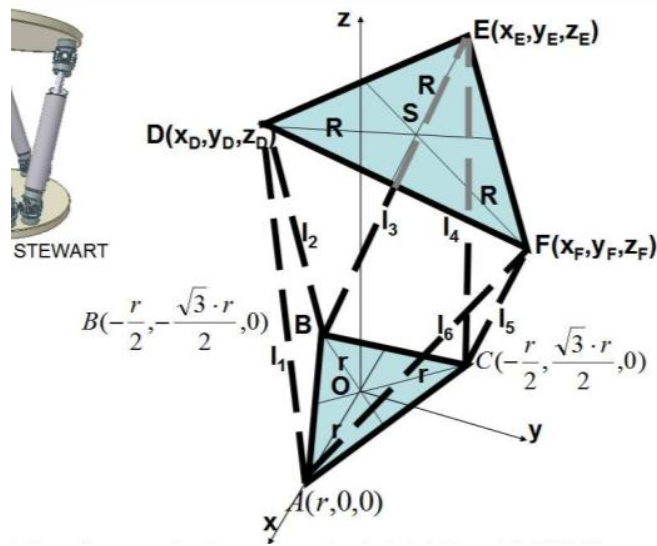

Fig. 10 A simple theoretical model

In figure 11 is determined the position parameters (spatial Cartesian coordinates) for fixed points $\mathrm{A}, \mathrm{B}, \mathrm{C}$. For point $\mathrm{A}$ is obtained $\mathrm{x}=\mathrm{r}$ and $\mathrm{y}=\mathrm{z}=0$. For point $\mathrm{B}$ using relations (7) and to determine the coordinates of $\mathrm{C}$ are considered system (8).

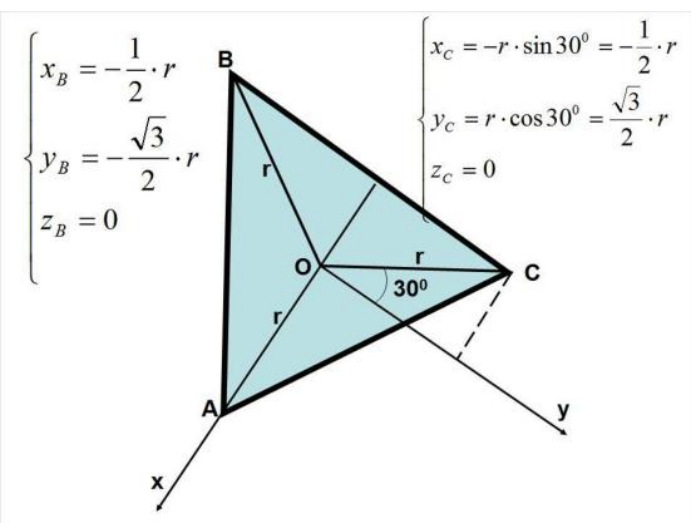

Fig. 11 Determining the fixed shelf

$$
\left\{\begin{array}{l}
x_{B}=-\frac{1}{2} \cdot r \\
y_{B}=-\frac{\sqrt{3}}{2} \cdot r \\
z_{B}=0
\end{array}\right.
$$




$$
\left\{\begin{array}{l}
x_{C}=-r \cdot \sin 30^{\circ}=-\frac{1}{2} \cdot r \\
y_{C}=r \cdot \cos 30^{\circ}=\frac{\sqrt{3}}{2} \cdot r \\
z_{C}=0
\end{array}\right.
$$

For mobile platform DEF (see Figure 12) can write equations (9). Basically were written distances between the peaks of the triangle DEF (pairwise) Cartesian coordinate space.

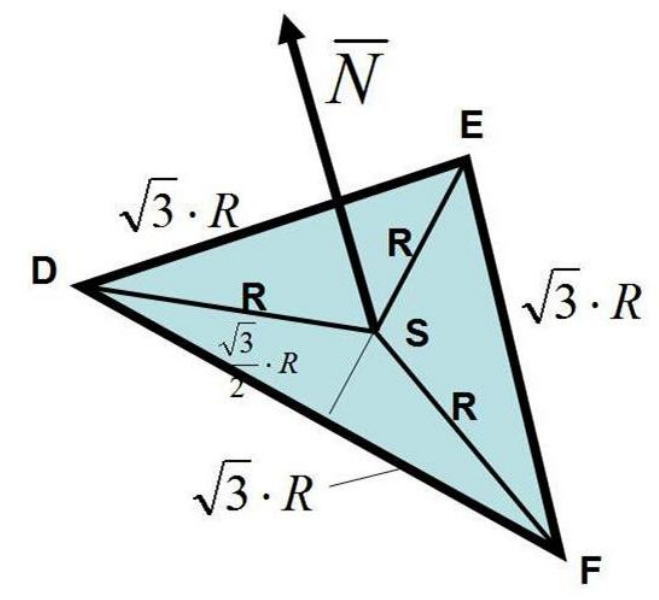

Fig. 12 Determining the mobile shelf

$$
\left\{\begin{array}{l}
\left(x_{D}-x_{F}\right)^{2}+\left(y_{D}-y_{F}\right)^{2}+\left(z_{D}-z_{F}\right)^{2}=3 \cdot R^{2} \\
\left(x_{D}-x_{E}\right)^{2}+\left(y_{D}-y_{E}\right)^{2}+\left(z_{D}-z_{E}\right)^{2}=3 \cdot R^{2} \\
\left(x_{E}-x_{F}\right)^{2}+\left(y_{E}-y_{F}\right)^{2}+\left(z_{E}-z_{F}\right)^{2}=3 \cdot R^{2}
\end{array}\right.
$$

The process was repeated but this time there were writing distances between mobile triangle center $\mathrm{S}$, and each vertex of the triangle DEF (eq. system 10).

$$
\left\{\begin{array}{l}
\left(x_{D}-x_{S}\right)^{2}+\left(y_{D}-y_{S}\right)^{2}+\left(z_{D}-z_{S}\right)^{2}=R^{2} \\
\left(x_{E}-x_{S}\right)^{2}+\left(y_{E}-y_{S}\right)^{2}+\left(z_{E}-z_{S}\right)^{2}=R^{2} \\
\left(x_{F}-x_{S}\right)^{2}+\left(y_{F}-y_{S}\right)^{2}+\left(z_{F}-z_{S}\right)^{2}=R^{2}
\end{array}\right.
$$

Write now DEF plan equation under general form (11), where D is any point of the plan, $\mathrm{S}$ is a particular (central) of the plane and the vector $\mathrm{N}$ is the vector perpendicular to the plan, considered in specifically chosen point $\mathrm{S}$.

The geometrical parameters (scalar) of position $(\alpha, \beta, \gamma)$ of the vector $\mathrm{N}$ are known.

The general equation of a plan say that any right line of plan scalar multiplied by the vector $\mathrm{N}$ (perpendicular on the plan) generates the product 0 . 


$$
\overline{D S} \cdot \bar{N}=0
$$

Will be assigned successive to the point $\mathrm{D}$ the values $\mathrm{D}, \mathrm{E}, \mathrm{F}$, and plan equation (11) written scalar will receive forms (12).

$$
\left\{\begin{array}{l}
\left(x_{D}-x_{S}\right) \cdot \alpha+\left(y_{D}-y_{S}\right) \cdot \beta+\left(z_{D}-z_{S}\right) \cdot \gamma=0 \\
\left(x_{E}-x_{S}\right) \cdot \alpha+\left(y_{E}-y_{S}\right) \cdot \beta+\left(z_{E}-z_{S}\right) \cdot \gamma=0 \\
\left(x_{F}-x_{S}\right) \cdot \alpha+\left(y_{F}-y_{S}\right) \cdot \beta+\left(z_{F}-z_{S}\right) \cdot \gamma=0
\end{array}\right.
$$

Scalar parameters $x S, y S, z S, \alpha, \beta, \gamma$ are known. With systems (12) and (10) can be determined immediately scalar parameters of a mobile point on the circle, choosing the initial determination of point $\mathrm{D}$, for example. Must this point be known (positioned) at least through one of its coordinates. Suppose for example ZD coordinate known (known propensity of mobile plan by $\alpha, \beta, \gamma$, one know where the focus $\mathrm{S}$ must be, knowing $\mathrm{xS}, \mathrm{yS}, \mathrm{zS}$, but must be known and $\mathrm{zD}$ height of a point on the mobile circle).

Determine then the other two scalar coordinates XD and yD. One use the system (13) formed by the first relation of the system (12) and by the first equation of the system (10).

$$
\left\{\begin{array}{l}
\left(x_{D}-x_{S}\right) \cdot \alpha+\left(y_{D}-y_{S}\right) \cdot \beta=\left(z_{S}-z_{D}\right) \cdot \gamma \\
\left(x_{D}-x_{S}\right)^{2}+\left(y_{D}-y_{S}\right)^{2}=R^{2}-\left(z_{D}-z_{S}\right)^{2}
\end{array}\right.
$$

To solve (13) are introduced notations (14).

$$
\left\{\begin{array}{l}
x=x_{D}-x_{S} \\
y=y_{D}-y_{S} \\
\theta=\left(z_{S}-z_{D}\right) \cdot \gamma \\
L^{2}=R^{2}-\left(z_{D}-z_{S}\right)^{2}
\end{array}\right.
$$

From (13) with the annotations (14) obtains the system (15), which is solved sequentially by relations (16) which leads to an equation grade 2 with unknown y, which solution is given by the first two relations of the system (17), while the third relation of the system (17) calculates $x$.

$$
\left\{\begin{array}{l}
\alpha \cdot x+\beta \cdot y=\theta \\
x^{2}+y^{2}=L^{2}
\end{array}\right.
$$




$$
\begin{aligned}
& \left\{\begin{array}{l}
x=\frac{\theta-\beta \cdot y}{\alpha} \quad x^{2}=\frac{\theta^{2}+\beta^{2} \cdot y^{2}-2 \cdot \theta \cdot \beta \cdot y}{\alpha^{2}} \\
\theta^{2}+\beta^{2} \cdot y^{2}-2 \cdot \theta \cdot \beta \cdot y+\alpha^{2} \cdot y^{2}=\alpha^{2} \cdot L^{2} \\
\left(\alpha^{2}+\beta^{2}\right) \cdot y^{2}-2 \cdot \theta \cdot \beta \cdot y-\left(\alpha^{2} \cdot L^{2}-\theta^{2}\right)=0
\end{array}\right. \\
& \left\{\begin{array}{l}
y_{1,2}=\frac{\theta \cdot \beta \pm \sqrt{\theta^{2} \cdot \beta^{2}+\left(\alpha^{2}+\beta^{2}\right) \cdot\left(\alpha^{2} \cdot L^{2}-\theta^{2}\right)}}{\alpha^{2}+\beta^{2}} \\
y_{1,2}=\frac{\theta \cdot \beta \pm \alpha \cdot \sqrt{\left(\alpha^{2}+\beta^{2}\right) \cdot L^{2}-\theta^{2}}}{\alpha^{2}+\beta^{2}} \\
x_{1,2}=\frac{\theta-\beta \cdot y}{\alpha}=\frac{\theta}{\alpha}-\frac{\beta}{\alpha} \cdot y_{1,2}
\end{array}\right.
\end{aligned}
$$

For proper positioning of the point $D$ is chosen initially negative solution (if it does not correspond to the positive solution will reelect). This gives scalar parameters of point $D$ (relation 18).

$$
\begin{cases}y=\frac{\theta \cdot \beta-\alpha \cdot \sqrt{\left(\alpha^{2}+\beta^{2}\right) \cdot L^{2}-\theta^{2}}}{\alpha^{2}+\beta^{2}} & y_{D}=y+y_{S} \\ x=\frac{\theta-\beta \cdot y}{\alpha}=\frac{\theta}{\alpha}-\frac{\beta}{\alpha} \cdot y & x_{D}=x+x_{S} \\ \Rightarrow D\left(x_{D}, y_{D}, z_{D}\right) & \end{cases}
$$

From $(12,10,9)$ are selected the equations with that one write the system (19), so as to have the only unknown the scalar coordinates of the point $\mathrm{E}$, namely: $\mathrm{xE}, \mathrm{yE}, \mathrm{zE}$.

$$
\left\{\begin{array}{l}
\left(x_{E}-x_{S}\right) \cdot \alpha+\left(y_{E}-y_{S}\right) \cdot \beta+\left(z_{E}-z_{S}\right) \cdot \gamma=0 \\
\left(x_{E}-x_{S}\right)^{2}+\left(y_{E}-y_{S}\right)^{2}+\left(z_{E}-z_{S}\right)^{2}=R^{2} \\
\left(x_{E}-x_{D}\right)^{2}+\left(y_{E}-y_{D}\right)^{2}+\left(z_{E}-z_{D}\right)^{2}=3 \cdot R^{2}
\end{array}\right.
$$

To solve the system (19) must be linearized. One squares the last two relations system (19) and subtract the second from the third. Third relationship is obtained in the system (20) which is arranged at a more convenient form trapped in the system (21) together with the first relation from the system (19) also ordered correspondingly.

$$
\left\{\begin{array}{l}
x_{E}^{2}+x_{S}^{2}-2 \cdot x_{S} \cdot x_{E}+y_{E}^{2}+y_{S}^{2}-2 \cdot y_{S} \cdot y_{E}+z_{E}^{2}+z_{S}^{2}-2 \cdot z_{S} \cdot z_{E}=R^{2} \\
x_{E}^{2}+x_{D}^{2}-2 \cdot x_{D} \cdot x_{E}+y_{E}^{2}+y_{D}^{2}-2 \cdot y_{D} \cdot y_{E}+z_{E}^{2}+z_{D}^{2}-2 \cdot z_{D} \cdot z_{E}=3 \cdot R^{2} \\
-------------------------------------- \\
x_{D}^{2}-x_{S}^{2}+2 \cdot\left(x_{S}-x_{D}\right) \cdot x_{E}+y_{D}^{2}-y_{S}^{2}+2 \cdot\left(y_{S}-y_{D}\right) \cdot y_{E}+z_{D}^{2}-z_{S}^{2}+ \\
+2 \cdot\left(z_{S}-z_{D}\right) \cdot z_{E}=2 \cdot R^{2}
\end{array}\right.
$$




$$
\left\{\begin{array}{l}
2 \cdot\left(x_{S}-x_{D}\right) \cdot x_{E}+2 \cdot\left(y_{S}-y_{D}\right) \cdot y_{E}+2 \cdot\left(z_{S}-z_{D}\right) \cdot z_{E}= \\
=2 \cdot R^{2}+x_{S}^{2}+y_{S}^{2}+z_{S}^{2}-x_{D}^{2}-y_{D}^{2}-z_{D}^{2} \\
\alpha \cdot x_{E}+\beta \cdot y_{E}+\gamma \cdot z_{E}=\alpha \cdot x_{S}+\beta \cdot y_{S}+\gamma \cdot z_{S}
\end{array}\right.
$$

From the second relation of the system (21) are explained zE (see expression 22), which is then introduced into the first relation of the system (21) thereby eliminating zE parameter, and to give expression (23) linearly with $\mathrm{yE}$ in relation of $\mathrm{xE}$, where the coefficients $\mathrm{k} 1, \mathrm{k} 2$, are determined by the relationships of the system (24).

$$
\begin{gathered}
z_{E}=\frac{\alpha}{\gamma} \cdot x_{S}+\frac{\beta}{\gamma} \cdot y_{S}+z_{S}-\frac{\alpha}{\gamma} \cdot x_{E}-\frac{\beta}{\gamma} \cdot y_{E} \\
y_{E}=k_{1}+k_{2} \cdot x_{E} \\
k_{1}=\left[2 \cdot R^{2}+x_{S}^{2}+y_{S}^{2}+z_{S}^{2}-x_{D}^{2}-y_{D}^{2}-z_{D}^{2}-2 \cdot\left(z_{S}-z_{D}\right) \cdot \frac{\alpha}{\gamma} \cdot x_{S}-\right. \\
\left.-2 \cdot\left(z_{S}-z_{D}\right) \cdot \frac{\beta}{\gamma} \cdot y_{S}-2 \cdot\left(z_{S}-z_{D}\right) \cdot z_{S}\right]:\left[2 \cdot\left(y_{S}-y_{D}\right)-2 \cdot\left(z_{S}-z_{D}\right) \cdot \frac{\beta}{\gamma}\right] \\
k_{2}=\frac{\left(x_{D}-x_{S}\right)+\left(z_{S}-z_{D}\right) \cdot \frac{\alpha}{\gamma}}{\left(y_{S}-y_{D}\right)-\left(z_{S}-z_{D}\right) \cdot \frac{\beta}{\gamma}}
\end{gathered}
$$

Replace now yE given by the relation (23) in the expression (22) and thus obtains a second linear relationship between parameters $\mathrm{zE}$ and $\mathrm{xE}$ (equation 25), whose coefficients $\mathrm{k} 3, \mathrm{k} 4$, are given by the system (26).

$$
\begin{aligned}
& z_{E}=k_{3}-k_{4} \cdot x_{E} \\
& \left\{\begin{array}{l}
k_{3}=\frac{\alpha}{\gamma} \cdot x_{S}+\frac{\beta}{\gamma} \cdot y_{S}+z_{S}-\frac{\beta}{\gamma} \cdot k_{1} \\
k_{4}=\frac{\alpha}{\gamma}+\frac{\beta}{\gamma} \cdot k_{2}
\end{array}\right.
\end{aligned}
$$

Relationships (23) and (25) are included simultaneously into the first relationship of the system (20) and in this mode one obtains an equation grade 2 in $\mathrm{xE}$ (expression 27), which is allocated to the form (28).

$$
\begin{aligned}
& \quad x_{E}^{2}-2 \cdot x_{S} \cdot x_{E}+\left(k_{1}+k_{2} \cdot x_{E}\right)^{2}-2 \cdot y_{S} \cdot\left(k_{1}+k_{2} \cdot x_{E}\right)+ \\
& \quad+\left(k_{3}-k_{4} \cdot x_{E}\right)^{2}-2 \cdot z_{S} \cdot\left(k_{3}-k_{4} \cdot x_{E}\right)= \\
& \quad=R^{2}-x_{S}^{2}-y_{S}^{2}-z_{S}^{2} \\
& \left(1+k_{2}^{2}+k_{4}^{2}\right) \cdot x_{E}^{2}-2 \cdot\left(x_{S}-k_{1} \cdot k_{2}+k_{2} \cdot y_{S}+k_{3} \cdot k_{4}\right) \cdot x_{E}+ \\
& +k_{1}^{2}-2 \cdot k_{1} \cdot y_{S}+k_{3}^{2}-2 \cdot k_{3} \cdot z_{S}-R^{2}+x_{S}^{2}+y_{S}^{2}+z_{S}^{2}=0
\end{aligned}
$$


We denote now the coefficients of equation (28) of second degree in $\mathrm{xE}$, with a1, b1, c1, (see relation 29).

$$
\left\{\begin{array}{l}
a_{1}=1+k_{2}^{2}+k_{4}^{2} \\
b_{1} \equiv-\frac{b}{2}=x_{S}-k_{1} \cdot k_{2}+k_{2} \cdot y_{S}+k_{3} \cdot k_{4} \\
c_{1}=k_{1}^{2}-2 \cdot k_{1} \cdot y_{S}+k_{3}^{2}-2 \cdot k_{3} \cdot z_{S}-R^{2}+x_{S}^{2}+y_{S}^{2}+z_{S}^{2}
\end{array}\right.
$$

Equation (28) takes the simplified form (30) which supports real solutions (31).

$$
\begin{aligned}
& a_{1} \cdot x_{E}^{2}-2 \cdot b_{1} \cdot x_{E}+c_{1}=0 \\
& x_{E_{1,2}}=\frac{b_{1} \pm \sqrt{b_{1}^{2}-a_{1} \cdot c_{1}}}{a_{1}}
\end{aligned}
$$

Choose the appropriate solution (the plus or the minus). Perhaps the solution would be negative.

One writes all scalar parameters of the point $\mathrm{E}$, by relations (32).

$$
\left\{\begin{array}{l}
x_{E}=\frac{b_{1}}{a_{1}}-\sqrt{\left(\frac{b_{1}}{a_{1}}\right)^{2}-\frac{c_{1}}{a_{1}}} \\
y_{E}=k_{1}+k_{2} \cdot x_{E} \\
z_{E}=k_{3}-k_{4} \cdot x_{E}
\end{array}\right.
$$

Now we have to determine Cartesian coordinates (rectangular, scalar) of mobile point F. From initial systems $(12,10,9)$ we can choose to use four relationships (one of 12, one of 10, and two from 9), relations with that one writes the system (33).

$$
\left\{\begin{array}{l}
\left(x_{F}-x_{S}\right) \cdot \alpha+\left(y_{F}-y_{S}\right) \cdot \beta+\left(z_{F}-z_{S}\right) \cdot \gamma=0 \\
\left(x_{F}-x_{S}\right)^{2}+\left(y_{F}-y_{S}\right)^{2}+\left(z_{F}-z_{S}\right)^{2}=R^{2} \\
\left(x_{F}-x_{D}\right)^{2}+\left(y_{F}-y_{D}\right)^{2}+\left(z_{F}-z_{D}\right)^{2}=3 \cdot R^{2} \\
\left(x_{F}-x_{E}\right)^{2}+\left(y_{F}-y_{E}\right)^{2}+\left(z_{F}-z_{E}\right)^{2}=3 \cdot R^{2}
\end{array}\right.
$$

The squared last two relations of the system (33) as expressions obtained (34) gather resulting equation (35), which is then arranged conveniently to the final form (36). 


$$
\begin{aligned}
& \left\{\begin{array}{l}
x_{F}^{2}+x_{D}^{2}-2 \cdot x_{D} \cdot x_{F}+y_{F}^{2}+y_{D}^{2}- \\
-2 \cdot y_{D} \cdot y_{F}+z_{F}^{2}+z_{D}^{2}-2 \cdot z_{D} \cdot z_{F}=3 \cdot R^{2} \\
x_{F}^{2}+x_{E}^{2}-2 \cdot x_{E} \cdot x_{F}+y_{F}^{2}+y_{E}^{2}- \\
-2 \cdot y_{E} \cdot y_{F}+z_{F}^{2}+z_{E}^{2}-2 \cdot z_{E} \cdot z_{F}=3 \cdot R^{2}
\end{array}\right. \\
& x_{D}^{2}-x_{E}^{2}+2 \cdot\left(x_{E}-x_{D}\right) \cdot x_{F}+y_{D}^{2}-y_{E}^{2}+ \\
& +2 \cdot\left(y_{E}-y_{D}\right) \cdot y_{F}+z_{D}^{2}-z_{E}^{2}+ \\
& +2 \cdot\left(z_{E}-z_{D}\right) \cdot z_{F}=0 \\
& 2 \cdot\left(x_{E}-x_{D}\right) \cdot x_{F}+2 \cdot\left(y_{E}-y_{D}\right) \cdot y_{F}+ \\
& +2 \cdot\left(z_{E}-z_{D}\right) \cdot z_{F}= \\
& =x_{E}^{2}-x_{D}^{2}+y_{E}^{2}-y_{D}^{2}+z_{E}^{2}-z_{D}^{2}
\end{aligned}
$$

Repeat all for the couple of equations the second and the third of the system of equations (33); one obtains the two equations system (37), which added produce the expression (38) that are arranged conveniently in the expression (39).

$$
\begin{aligned}
& \left\{\begin{array}{l}
x_{F}^{2}+x_{S}^{2}-2 \cdot x_{S} \cdot x_{F}+y_{F}^{2}+y_{S}^{2}- \\
-2 \cdot y_{S} \cdot y_{F}+z_{F}^{2}+z_{S}^{2}-2 \cdot z_{S} \cdot z_{F}=R^{2} \\
x_{F}^{2}+x_{D}^{2}-2 \cdot x_{D} \cdot x_{F}+y_{F}^{2}+y_{D}^{2}- \\
-2 \cdot y_{D} \cdot y_{F}+z_{F}^{2}+z_{D}^{2}-2 \cdot z_{D} \cdot z_{F}=3 \cdot R^{2}
\end{array}\right. \\
& x_{D}^{2}-x_{S}^{2}+2 \cdot\left(x_{S}-x_{D}\right) \cdot x_{F}+y_{D}^{2}-y_{S}^{2}+ \\
& +2 \cdot\left(y_{S}-y_{D}\right) \cdot y_{F}+z_{D}^{2}-z_{S}^{2}+ \\
& +2 \cdot\left(z_{S}-z_{D}\right) \cdot z_{F}=2 \cdot R^{2} \\
& 2 \cdot\left(x_{S}-x_{D}\right) \cdot x_{F}+2 \cdot\left(y_{S}-y_{D}\right) \cdot y_{F}+ \\
& +2 \cdot\left(z_{S}-z_{D}\right) \cdot z_{F}= \\
& =2 \cdot R^{2}+x_{S}^{2}-x_{D}^{2}+y_{S}^{2}-y_{D}^{2}+z_{S}^{2}-z_{D}^{2}
\end{aligned}
$$

It retains the linear system (40) of three equations with three unknowns, where the three equations are (36), (39) and the first relation of the system (33). 


$$
\left\{\begin{array}{l}
2\left(x_{E}-x_{D}\right) x_{F}+2\left(y_{E}-y_{D}\right) y_{F}+2\left(z_{E}-z_{D}\right) z_{F}= \\
=x_{E}^{2}-x_{D}^{2}+y_{E}^{2}-y_{D}^{2}+z_{E}^{2}-z_{D}^{2} \\
2\left(x_{S}-x_{D}\right) x_{F}+2\left(y_{S}-y_{D}\right) y_{F}+2\left(z_{S}-z_{D}\right) z_{F}= \\
=2 R^{2}+x_{S}^{2}-x_{D}^{2}+y_{S}^{2}-y_{D}^{2}+z_{S}^{2}-z_{D}^{2} \\
\alpha \cdot x_{F}+\beta \cdot y_{F}+\gamma \cdot z_{F}=\alpha \cdot x_{S}+\beta \cdot y_{S}+\gamma \cdot z_{S}
\end{array}\right.
$$

The system (40) is written as classical (41).

$$
\left\{\begin{array}{l}
a_{11} \cdot x_{F}+a_{12} \cdot y_{F}+a_{13} \cdot z_{F}=b_{1} \\
a_{21} \cdot x_{F}+a_{22} \cdot y_{F}+a_{23} \cdot z_{F}=b_{2} \\
a_{31} \cdot x_{F}+a_{32} \cdot y_{F}+a_{33} \cdot z_{F}=b_{3}
\end{array}\right.
$$

The coefficients of the system (41) are determined by the relations (42).

$$
\left\{\begin{array}{l}
a_{11}=2 \cdot\left(x_{E}-x_{D}\right) ; \\
a_{12}=2 \cdot\left(y_{E}-y_{D}\right) ; \\
a_{13}=2 \cdot\left(z_{E}-z_{D}\right) ; \\
b_{1}=x_{E}^{2}-x_{D}^{2}+y_{E}^{2}-y_{D}^{2}+z_{E}^{2}-z_{D}^{2} ; \\
a_{21}=2 \cdot\left(x_{S}-x_{D}\right) ; \\
a_{22}=2 \cdot\left(y_{S}-y_{D}\right) ; \\
a_{23}=2 \cdot\left(z_{S}-z_{D}\right) ; \\
b_{2}=2 \cdot R^{2}+x_{S}^{2}-x_{D}^{2}+y_{S}^{2}-y_{D}^{2}+z_{S}^{2}-z_{D}^{2} \\
a_{31}=\alpha ; \quad a_{32}=\beta ; \quad a_{33}=\gamma ; \\
b_{3}=\alpha \cdot x_{S}+\beta \cdot y_{S}+\gamma \cdot z_{S}
\end{array}\right.
$$

Determinants system (41) is determined by relations (43-46).

$$
\begin{aligned}
& \Delta=\left|\begin{array}{ccc}
a_{11} & a_{12} & a_{13} \\
a_{21} & a_{22} & a_{23} \\
a_{31} & a_{32} & a_{33}
\end{array}\right|= \\
& =a_{11} \cdot\left(a_{22} \cdot a_{33}-a_{23} \cdot a_{32}\right)+ \\
& +a_{12} \cdot\left(a_{23} \cdot a_{31}-a_{21} \cdot a_{33}\right)+ \\
& +a_{13} \cdot\left(a_{21} \cdot a_{32}-a_{22} \cdot a_{31}\right)
\end{aligned}
$$




$$
\begin{aligned}
& \Delta_{x}=\left|\begin{array}{lll}
b_{1} & a_{12} & a_{13} \\
b_{2} & a_{22} & a_{23} \\
b_{3} & a_{32} & a_{33}
\end{array}\right|= \\
& =b_{1} \cdot\left(a_{22} \cdot a_{33}-a_{23} \cdot a_{32}\right)+ \\
& +a_{12} \cdot\left(a_{23} \cdot b_{3}-b_{2} \cdot a_{33}\right)+ \\
& +a_{13} \cdot\left(b_{2} \cdot a_{32}-a_{22} \cdot b_{3}\right) \\
& \Delta_{y}=\left|\begin{array}{lll}
a_{11} & b_{1} & a_{13} \\
a_{21} & b_{2} & a_{23} \\
a_{31} & b_{3} & a_{33}
\end{array}\right|= \\
& =a_{11} \cdot\left(b_{2} \cdot a_{33}-a_{23} \cdot b_{3}\right)+ \\
& +b_{1} \cdot\left(a_{23} \cdot a_{31}-a_{21} \cdot a_{33}\right)+ \\
& +a_{13} \cdot\left(a_{21} \cdot b_{3}-b_{2} \cdot a_{31}\right) \\
& \Delta_{z}=\left|\begin{array}{lll}
a_{11} & a_{12} & b_{1} \\
a_{21} & a_{22} & b_{2} \\
a_{31} & a_{32} & b_{3}
\end{array}\right|= \\
& =a_{11} \cdot\left(a_{22} \cdot b_{3}-b_{2} \cdot a_{32}\right)+ \\
& +a_{12} \cdot\left(b_{2} \cdot a_{31}-a_{21} \cdot b_{3}\right)+ \\
& +b_{1} \cdot\left(a_{21} \cdot a_{32}-a_{22} \cdot a_{31}\right)
\end{aligned}
$$

System solutions are given by relations (47).

$$
\left\{\begin{array}{l}
x_{F}=\frac{\Delta_{x}}{\Delta} \\
y_{F}=\frac{\Delta_{y}}{\Delta} \\
z_{F}=\frac{\Delta_{z}}{\Delta}
\end{array}\right.
$$

With the known coordinates of the points D, E, F, imposed by the choice of position of the DEF plan and point $\mathrm{D}$, one determines then the required length of the legs (see the relations $48)$. 


$$
\left\{\begin{array}{l}
l_{1}=\sqrt{\left(x_{D}-x_{A}\right)^{2}+\left(y_{D}-y_{A}\right)^{2}+\left(z_{D}-z_{A}\right)^{2}} \\
l_{2}=\sqrt{\left(x_{D}-x_{B}\right)^{2}+\left(y_{D}-y_{B}\right)^{2}+\left(z_{D}-z_{B}\right)^{2}} \\
l_{3}=\sqrt{\left(x_{E}-x_{B}\right)^{2}+\left(y_{E}-y_{B}\right)^{2}+\left(z_{E}-z_{B}\right)^{2}} \\
l_{4}=\sqrt{\left(x_{E}-x_{C}\right)^{2}+\left(y_{E}-y_{C}\right)^{2}+\left(z_{E}-z_{C}\right)^{2}} \\
l_{5}=\sqrt{\left(x_{F}-x_{C}\right)^{2}+\left(y_{F}-y_{C}\right)^{2}+\left(z_{F}-z_{C}\right)^{2}} \\
l_{6}=\sqrt{\left(x_{F}-x_{A}\right)^{2}+\left(y_{F}-y_{A}\right)^{2}+\left(z_{F}-z_{A}\right)^{2}}
\end{array}\right.
$$

\section{Determining the velocities}

One knows: $\dot{x}_{S}, \dot{y}_{S}, \dot{z}_{S}, \dot{\alpha}, \dot{\beta}, \dot{\gamma}, \dot{z}_{D}$. Choose the relations (13) writhed here as (49) to start.

$$
\left\{\begin{array}{l}
\left(x_{D}-x_{S}\right) \cdot \alpha+\left(y_{D}-y_{S}\right) \cdot \beta=\left(z_{S}-z_{D}\right) \cdot \gamma \\
\left(x_{D}-x_{S}\right)^{2}+\left(y_{D}-y_{S}\right)^{2}=R^{2}-\left(z_{D}-z_{S}\right)^{2}
\end{array}\right.
$$

Relationships (49) derived in function of time give the expressions (50).

$$
\left\{\begin{array}{l}
\left(\dot{x}_{D}-\dot{x}_{S}\right) \cdot \alpha+\left(x_{D}-x_{S}\right) \cdot \dot{\alpha}+\left(\dot{y}_{D}-\dot{y}_{S}\right) \cdot \beta+\left(y_{D}-y_{S}\right) \cdot \dot{\beta}= \\
=\left(\dot{z}_{S}-\dot{z}_{D}\right) \cdot \gamma+\left(z_{S}-z_{D}\right) \cdot \dot{\gamma} \\
2 \cdot\left(x_{D}-x_{S}\right) \cdot\left(\dot{x}_{D}-\dot{x}_{S}\right)+2 \cdot\left(y_{D}-y_{S}\right) \cdot\left(\dot{y}_{D}-\dot{y}_{S}\right)= \\
=-2 \cdot\left(z_{D}-z_{S}\right) \cdot\left(\dot{z}_{D}-\dot{z}_{S}\right)
\end{array}\right.
$$

These are arranged in the form (51). There was thus obtained a linear system of two equations in two unknowns, identified by (52).

$$
\left\{\begin{array}{l}
\alpha \cdot \dot{x}_{D}+\beta \cdot \dot{y}_{D}=\alpha \cdot \dot{x}_{S}-\left(x_{D}-x_{S}\right) \cdot \dot{\alpha}+\beta \cdot \dot{y}_{S}- \\
-\left(y_{D}-y_{S}\right) \cdot \dot{\beta}+\left(\dot{z}_{S}-\dot{z}_{D}\right) \cdot \gamma+\left(z_{S}-z_{D}\right) \cdot \dot{\gamma} \\
\left(x_{D}-x_{S}\right) \cdot \dot{x}_{D}+\left(y_{D}-y_{S}\right) \cdot \dot{y}_{D}=\left(x_{D}-x_{S}\right) \cdot \dot{x}_{S}+ \\
+\left(y_{D}-y_{S}\right) \cdot \dot{y}_{S}-\left(z_{D}-z_{S}\right) \cdot\left(\dot{z}_{D}-\dot{z}_{S}\right)
\end{array}\right.
$$




$$
\left\{\begin{array}{l}
a_{11} \cdot \dot{x}_{D}+a_{12} \cdot \dot{y}_{D}=b_{1} \\
a_{21} \cdot \dot{x}_{D}+a_{22} \cdot \dot{y}_{D}=b_{2} \\
a_{11}=\alpha ; \quad a_{12}=\beta ; \\
a_{21}=x_{D}-x_{S} ; \quad a_{22}=y_{D}-y_{S} ; \\
b_{1}=\alpha \cdot \dot{x}_{S}-\left(x_{D}-x_{S}\right) \cdot \dot{\alpha}+\beta \cdot \dot{y}_{S}- \\
-\left(y_{D}-y_{S}\right) \cdot \dot{\beta}+\left(\dot{z}_{S}-\dot{z}_{D}\right) \cdot \gamma+\left(z_{S}-z_{D}\right) \cdot \dot{\gamma} \\
b_{2}=\left(x_{D}-x_{S}\right) \cdot \dot{x}_{S}+\left(y_{D}-y_{S}\right) \cdot \dot{y}_{S}- \\
-\left(z_{D}-z_{S}\right) \cdot\left(\dot{z}_{D}-\dot{z}_{S}\right)
\end{array}\right.
$$

Determinant of the system (51-52) is written in equation (53).

$$
\begin{aligned}
& \Delta=\left|\begin{array}{ll}
a_{11} & a_{12} \\
a_{21} & a_{22}
\end{array}\right|=a_{11} \cdot a_{22}-a_{12} \cdot a_{21}= \\
& =\alpha \cdot\left(y_{D}-y_{S}\right)-\beta \cdot\left(x_{D}-x_{S}\right)
\end{aligned}
$$

One calculates $\Delta_{x 1}$ with the relation (54) and $\dot{x}_{D}$ by the expression (55).

$$
\begin{aligned}
& \Delta_{x 1}=\left|\begin{array}{ll}
b_{1} & a_{12} \\
b_{2} & a_{22}
\end{array}\right|=b_{1} \cdot a_{22}-a_{12} \cdot b_{2} \\
& \dot{x}_{D}=\frac{\Delta_{x 1}}{\Delta}
\end{aligned}
$$

One calculates $\Delta_{y 1}$ with the relation (56) and $\dot{y}_{D}$ by the expression (57).

$$
\begin{aligned}
\Delta_{y 1} & =\left|\begin{array}{ll}
a_{11} & b_{1} \\
a_{21} & b_{2}
\end{array}\right|=a_{11} \cdot b_{2}-b_{1} \cdot a_{21} \\
\dot{y}_{D} & =\frac{\Delta_{y 1}}{\Delta}
\end{aligned}
$$

It is still write system (58), which is derived in function of time and in this mode taking the form (59). 


$$
\begin{aligned}
& \left\{\begin{array}{l}
\left(x_{E}-x_{S}\right) \cdot \alpha+\left(y_{E}-y_{S}\right) \cdot \beta+\left(z_{E}-z_{S}\right) \cdot \gamma=0 \\
\left(x_{E}-x_{S}\right)^{2}+\left(y_{E}-y_{S}\right)^{2}+\left(z_{E}-z_{S}\right)^{2}=R^{2} \\
\left(x_{E}-x_{D}\right)^{2}+\left(y_{E}-y_{D}\right)^{2}+\left(z_{E}-z_{D}\right)^{2}=3 \cdot R^{2}
\end{array}\right. \\
& \left\{\begin{array}{l}
\left(\dot{x}_{E}-\dot{x}_{S}\right) \cdot \alpha+\left(x_{E}-x_{S}\right) \cdot \dot{\alpha}+\left(\dot{y}_{E}-\dot{y}_{S}\right) \cdot \beta+ \\
+\left(y_{E}-y_{S}\right) \cdot \dot{\beta}+\left(\dot{z}_{E}-\dot{z}_{S}\right) \cdot \gamma+\left(z_{E}-z_{S}\right) \cdot \dot{\gamma}=0 \\
2 \cdot\left(x_{E}-x_{S}\right) \cdot\left(\dot{x}_{E}-\dot{x}_{S}\right)+2 \cdot\left(y_{E}-y_{S}\right) \cdot\left(\dot{y}_{E}-\dot{y}_{S}\right)+ \\
+2 \cdot\left(z_{E}-z_{S}\right) \cdot\left(\dot{z}_{E}-\dot{z}_{S}\right)=0 \\
2 \cdot\left(x_{E}-x_{D}\right) \cdot\left(\dot{x}_{E}-\dot{x}_{D}\right)+2 \cdot\left(y_{E}-y_{D}\right) \cdot\left(\dot{y}_{E}-\dot{y}_{D}\right)+ \\
+2 \cdot\left(z_{E}-z_{D}\right) \cdot\left(\dot{z}_{E}-\dot{z}_{D}\right)=0
\end{array}\right.
\end{aligned}
$$

For solving the system (59) is allocated to the form (60), which is a linear system of three equations with three unknown level one, identified by formulas in the system (61).

$$
\begin{aligned}
& \left\{\begin{array}{l}
\alpha \cdot \dot{x}_{E}+\beta \cdot \dot{y}_{E}+\gamma \cdot \dot{z}_{E}=\alpha \cdot \dot{x}_{S}-\left(x_{E}-x_{S}\right) \cdot \dot{\alpha}+ \\
+\beta \cdot \dot{y}_{S}-\left(y_{E}-y_{S}\right) \cdot \dot{\beta}+\gamma \cdot \dot{z}_{S}-\left(z_{E}-z_{S}\right) \cdot \dot{\gamma} \\
\left(x_{E}-x_{S}\right) \cdot \dot{x}_{E}+\left(y_{E}-y_{S}\right) \cdot \dot{y}_{E}+\left(z_{E}-z_{S}\right) \cdot \dot{z}_{E}= \\
=\left(x_{E}-x_{S}\right) \cdot \dot{x}_{S}+\left(y_{E}-y_{S}\right) \cdot \dot{y}_{S}+\left(z_{E}-z_{S}\right) \cdot \dot{z}_{S} \\
\left(x_{E}-x_{D}\right) \cdot \dot{x}_{E}+\left(y_{E}-y_{D}\right) \cdot \dot{y}_{E}+\left(z_{E}-z_{D}\right) \cdot \dot{z}_{E}= \\
=\left(x_{E}-x_{D}\right) \cdot \dot{x}_{D}+\left(y_{E}-y_{D}\right) \cdot \dot{y}_{D}+\left(z_{E}-z_{D}\right) \cdot \dot{z}_{D}
\end{array}\right. \\
& \left\{\begin{array}{l}
c_{11} \cdot \dot{x}_{E}+c_{12} \cdot \dot{y}_{E}+c_{13} \cdot \dot{z}_{E}=c_{1} \\
c_{21} \cdot \dot{x}_{E}+c_{22} \cdot \dot{y}_{E}+c_{23} \cdot \dot{z}_{E}=c_{2} \\
c_{31} \cdot \dot{x}_{E}+c_{32} \cdot \dot{y}_{E}+c_{33} \cdot \dot{z}_{E}=c_{3} \\
c_{11}=\alpha ; \quad c_{12}=\beta ; \quad c_{13}=\gamma ; \\
c_{1}=\alpha \cdot \dot{x}_{S}-\left(x_{E}-x_{S}\right) \cdot \dot{\alpha}+\beta \cdot \dot{y}_{S}- \\
-\left(y_{E}-y_{S}\right) \cdot \dot{\beta}+\gamma \cdot \dot{z}_{S}-\left(z_{E}-z_{S}\right) \cdot \dot{\gamma} \\
c_{21}=x_{E}-x_{S} ; \quad c_{22}=y_{E}-y_{S} ; \quad c_{23}=z_{E}-z_{s} ; \\
c_{2}=\left(x_{E}-x_{S}\right) \cdot \dot{x}_{S}+\left(y_{E}-y_{S}\right) \cdot \dot{y}_{S}+\left(z_{E}-z_{S}\right) \cdot \dot{z}_{S}
\end{array}\right.
\end{aligned}
$$

The main determinant of the system (61) is given by relations (62). 


$$
\left\{\begin{array}{l}
\Delta^{(c)}=\left|\begin{array}{lll}
c_{11} & c_{12} & c_{13} \\
c_{21} & c_{22} & c_{23} \\
c_{31} & c_{32} & c_{33}
\end{array}\right|=c_{11} \cdot\left(c_{22} \cdot c_{33}-c_{23} \cdot c_{32}\right)- \\
-c_{12} \cdot\left(c_{21} \cdot c_{33}-c_{23} \cdot c_{31}\right)+c_{13} \cdot\left(c_{21} \cdot c_{32}-c_{22} \cdot c_{31}\right) \\
\Delta^{(c)}=\alpha \cdot\left[\left(y_{E}-y_{S}\right) \cdot\left(z_{E}-z_{D}\right)-\left(z_{E}-z_{S}\right) \cdot\left(y_{E}-y_{D}\right)\right]- \\
-\beta \cdot\left[\left(x_{E}-x_{S}\right) \cdot\left(z_{E}-z_{D}\right)-\left(z_{E}-z_{S}\right) \cdot\left(x_{E}-x_{D}\right)\right]+ \\
+\gamma \cdot\left[\left(x_{E}-x_{S}\right) \cdot\left(y_{E}-y_{D}\right)-\left(y_{E}-y_{S}\right) \cdot\left(x_{E}-x_{D}\right)\right]
\end{array}\right.
$$

Determinant first scalar speed is calculated with equation (63).

$$
\left\{\begin{array}{l}
\Delta_{x}^{(c)}=\left|\begin{array}{ccc}
c_{1} & c_{12} & c_{13} \\
c_{2} & c_{22} & c_{23} \\
c_{3} & c_{32} & c_{33}
\end{array}\right|=c_{1} \cdot\left(c_{22} \cdot c_{33}-c_{23} \cdot c_{32}\right)- \\
-c_{12} \cdot\left(c_{2} \cdot c_{33}-c_{23} \cdot c_{3}\right)+c_{13} \cdot\left(c_{2} \cdot c_{32}-c_{22} \cdot c_{3}\right)
\end{array}\right.
$$

First scalar velocity $\dot{x}_{E}$ is determined by the expression (64).

$$
\dot{x}_{E}=\frac{\Delta_{x}^{(c)}}{\Delta^{(c)}}
$$

The determinant of the second scalar speed is calculated by equation (65).

$$
\left\{\begin{array}{l}
\Delta_{y}^{(c)}=\left|\begin{array}{lll}
c_{11} & c_{1} & c_{13} \\
c_{21} & c_{2} & c_{23} \\
c_{31} & c_{3} & c_{33}
\end{array}\right|=c_{11} \cdot\left(c_{2} \cdot c_{33}-c_{23} \cdot c_{3}\right)- \\
-c_{1} \cdot\left(c_{21} \cdot c_{33}-c_{23} \cdot c_{31}\right)+c_{13} \cdot\left(c_{21} \cdot c_{3}-c_{2} \cdot c_{31}\right)
\end{array}\right.
$$

The second scalar velocity $\dot{y}_{E}$ is determined by the expression (66).

$$
\dot{y}_{E}=\frac{\Delta_{y}^{(c)}}{\Delta^{(c)}}
$$

The determinant of the third scalar speed is calculated with the relation (67).

$$
\left\{\begin{array}{l}
\Delta_{z}^{(c)}=\left|\begin{array}{ccc}
c_{11} & c_{12} & c_{1} \\
c_{21} & c_{22} & c_{2} \\
c_{31} & c_{32} & c_{3}
\end{array}\right|=c_{11} \cdot\left(c_{22} \cdot c_{3}-c_{2} \cdot c_{32}\right)- \\
-c_{12} \cdot\left(c_{21} \cdot c_{3}-c_{2} \cdot c_{31}\right)+c_{1} \cdot\left(c_{21} \cdot c_{32}-c_{22} \cdot c_{31}\right)
\end{array}\right.
$$

The third scalar velocity $\dot{z}_{E}$ is determined by the expression (68). 


$$
\dot{z}_{E}=\frac{\Delta_{z}^{(c)}}{\Delta^{(c)}}
$$

For scalar velocities of the last floating point $\mathrm{F}$ one starts from the positions system known (69), which is derived by time and results the system (70).

$$
\begin{gathered}
\left\{\begin{array}{l}
\left(x_{F}-x_{S}\right) \cdot \alpha+\left(y_{F}-y_{S}\right) \cdot \beta+\left(z_{F}-z_{S}\right) \cdot \gamma=0 \\
\left(x_{F}-x_{S}\right)^{2}+\left(y_{F}-y_{S}\right)^{2}+\left(z_{F}-z_{S}\right)^{2}=R^{2} \\
\left(x_{F}-x_{D}\right)^{2}+\left(y_{F}-y_{D}\right)^{2}+\left(z_{F}-z_{D}\right)^{2}=3 \cdot R^{2}
\end{array}\right. \\
\left\{\begin{array}{l}
\left(\dot{x}_{F}-\dot{x}_{S}\right) \cdot \alpha+\left(x_{F}-x_{S}\right) \cdot \dot{\alpha}+\left(\dot{y}_{F}-\dot{y}_{S}\right) \cdot \beta+ \\
+\left(y_{F}-y_{S}\right) \cdot \dot{\beta}+\left(\dot{z}_{F}-\dot{z}_{S}\right) \cdot \gamma+\left(z_{F}-z_{S}\right) \cdot \dot{\gamma}=0 \\
2 \cdot\left(x_{F}-x_{S}\right) \cdot\left(\dot{x}_{F}-\dot{x}_{S}\right)+2 \cdot\left(y_{F}-y_{S}\right) \cdot\left(\dot{y}_{F}-\dot{y}_{S}\right)+ \\
+2 \cdot\left(z_{F}-z_{S}\right) \cdot\left(\dot{z}_{F}-\dot{z}_{S}\right)=0 \\
2 \cdot\left(x_{F}-x_{D}\right) \cdot\left(\dot{x}_{F}-\dot{x}_{D}\right)+2 \cdot\left(y_{F}-y_{D}\right) \cdot\left(\dot{y}_{F}-\dot{y}_{D}\right)+ \\
+2 \cdot\left(z_{F}-z_{D}\right) \cdot\left(\dot{z}_{F}-\dot{z}_{D}\right)=0
\end{array}\right.
\end{gathered}
$$

The system (70) is arranged in the form (71) which is a linear system of three equations with three unknowns first degree, whose equations are identified by (72) and whose parameters are written in the form (73).

$$
\begin{gathered}
\left\{\begin{array}{l}
\alpha \cdot \dot{x}_{F}+\beta \cdot \dot{y}_{F}+\gamma \cdot \dot{z}_{F}=\alpha \cdot \dot{x}_{S}+\beta \cdot \dot{y}_{S}+\gamma \cdot \dot{z}_{S}- \\
-\left(x_{F}-x_{S}\right) \cdot \dot{\alpha}-\left(y_{F}-y_{S}\right) \cdot \dot{\beta}-\left(z_{F}-z_{S}\right) \cdot \dot{\gamma} \\
\left(x_{F}-x_{S}\right) \cdot \dot{x}_{F}+\left(y_{F}-y_{S}\right) \cdot \dot{y}_{F}+\left(z_{F}-z_{S}\right) \cdot \dot{z}_{F}= \\
=\left(x_{F}-x_{S}\right) \cdot \dot{x}_{S}+\left(y_{F}-y_{S}\right) \cdot \dot{y}_{S}+\left(z_{F}-z_{S}\right) \cdot \dot{z}_{S} \\
\left(x_{F}-x_{D}\right) \cdot \dot{x}_{F}+\left(y_{F}-y_{D}\right) \cdot \dot{y}_{F}+\left(z_{F}-z_{D}\right) \cdot \dot{z}_{F}= \\
=\left(x_{F}-x_{D}\right) \cdot \dot{x}_{D}+\left(y_{F}-y_{D}\right) \cdot \dot{y}_{D}+\left(z_{F}-z_{D}\right) \cdot \dot{z}_{D}
\end{array}\right. \\
\left\{\begin{array}{l}
d_{11} \cdot \dot{x}_{F}+d_{12} \cdot \dot{y}_{F}+d_{13} \cdot \dot{z}_{F}=d_{1} \\
d_{21} \cdot \dot{x}_{F}+d_{22} \cdot \dot{y}_{F}+d_{23} \cdot \dot{z}_{F}=d_{2} \\
d_{31} \cdot \dot{x}_{F}+d_{32} \cdot \dot{y}_{F}+d_{33} \cdot \dot{z}_{F}=d_{3}
\end{array}\right.
\end{gathered}
$$




$$
\left\{\begin{array}{l}
d_{11}=\alpha ; \quad d_{12}=\beta ; \quad d_{13}=\gamma ; \\
d_{1}=\alpha \cdot \dot{x}_{S}+\beta \cdot \dot{y}_{S}+\gamma \cdot \dot{z}_{S}-\left(x_{F}-x_{S}\right) \cdot \dot{\alpha}- \\
-\left(y_{F}-y_{S}\right) \cdot \dot{\beta}-\left(z_{F}-z_{S}\right) \cdot \dot{\gamma} ; \\
d_{21}=x_{F}-x_{S} ; \quad d_{22}=y_{F}-y_{S} ; \quad d_{23}=z_{F}-z_{S} ; \\
d_{2}=\left(x_{F}-x_{S}\right) \cdot \dot{x}_{S}+\left(y_{F}-y_{S}\right) \cdot \dot{y}_{S}+\left(z_{F}-z_{S}\right) \cdot \dot{z}_{S} \\
d_{31}=x_{F}-x_{D} ; \quad d_{32}=y_{F}-y_{D} ; \quad d_{33}=z_{F}-z_{D} ; \\
d_{3}=\left(x_{F}-x_{D}\right) \cdot \dot{x}_{D}+\left(y_{F}-y_{D}\right) \cdot \dot{y}_{D}+\left(z_{F}-z_{D}\right) \cdot \dot{z}_{D}
\end{array}\right.
$$

The four determinants of the system are written in equations (74-77), the primary determinant being given even by relation (74).

$$
\left\{\begin{array}{l}
\Delta^{(d)}=\left|\begin{array}{lll}
d_{11} & d_{12} & d_{13} \\
d_{21} & d_{22} & d_{23} \\
d_{31} & d_{32} & d_{33}
\end{array}\right|=d_{11} \cdot\left(d_{22} \cdot d_{33}-d_{23} \cdot d_{32}\right)- \\
-d_{12} \cdot\left(d_{21} \cdot d_{33}-d_{23} \cdot d_{31}\right)+d_{13} \cdot\left(d_{21} \cdot d_{32}-d_{22} \cdot d_{31}\right)
\end{array}\right.
$$

$$
\left\{\begin{array}{l}
\Delta_{x}^{(d)}=\left|\begin{array}{ccc}
d_{1} & d_{12} & d_{13} \\
d_{2} & d_{22} & d_{23} \\
d_{3} & d_{32} & d_{33}
\end{array}\right|=d_{1} \cdot\left(d_{22} \cdot d_{33}-d_{23} \cdot d_{32}\right)- \\
-d_{12} \cdot\left(d_{2} \cdot d_{33}-d_{3} \cdot d_{23}\right)+d_{13} \cdot\left(d_{2} \cdot d_{32}-d_{3} \cdot d_{22}\right)
\end{array}\right.
$$

$$
\left\{\begin{array}{l}
\Delta_{y}^{(d)}=\left|\begin{array}{ccc}
d_{11} & d_{1} & d_{13} \\
d_{21} & d_{2} & d_{23} \\
d_{31} & d_{3} & d_{33}
\end{array}\right|=d_{11} \cdot\left(d_{2} \cdot d_{33}-d_{3} \cdot d_{23}\right)- \\
-d_{1} \cdot\left(d_{21} \cdot d_{33}-d_{23} \cdot d_{31}\right)+d_{13} \cdot\left(d_{21} \cdot d_{3}-d_{2} \cdot d_{31}\right)
\end{array}\right.
$$

$$
\left\{\begin{array}{l}
\Delta_{z}^{(d)}=\left|\begin{array}{ccc}
d_{11} & d_{12} & d_{1} \\
d_{21} & d_{22} & d_{2} \\
d_{31} & d_{32} & d_{3}
\end{array}\right|=d_{11} \cdot\left(d_{22} \cdot d_{3}-d_{2} \cdot d_{32}\right)- \\
-d_{12} \cdot\left(d_{21} \cdot d_{3}-d_{2} \cdot d_{31}\right)+d_{1} \cdot\left(d_{21} \cdot d_{32}-d_{22} \cdot d_{31}\right)
\end{array}\right.
$$

Gear system scalar solutions are obtained by using the relations (78).

$$
\left\{\dot{x}_{F}=\frac{\Delta_{x}^{(d)}}{\Delta^{(d)}} ; \quad \dot{y}_{F}=\frac{\Delta_{y}^{(d)}}{\Delta^{(d)}} ; \quad \dot{z}_{F}=\frac{\Delta_{z}^{(d)}}{\Delta^{(d)}} ;\right.
$$


Mobile plan speeds (upper) being determined, one can move to the final stage in which will be determined linear speeds of the six engines couplers (the translation). Must to write first relations of positions (79).

$$
\left\{\begin{array}{l}
l_{1}^{2}=\left(x_{D}-x_{A}\right)^{2}+\left(y_{D}-y_{A}\right)^{2}+\left(z_{D}-z_{A}\right)^{2} \\
l_{2}^{2}=\left(x_{D}-x_{B}\right)^{2}+\left(y_{D}-y_{B}\right)^{2}+\left(z_{D}-z_{B}\right)^{2} \\
l_{3}^{2}=\left(x_{E}-x_{B}\right)^{2}+\left(y_{E}-y_{B}\right)^{2}+\left(z_{E}-z_{B}\right)^{2} \\
l_{4}^{2}=\left(x_{E}-x_{C}\right)^{2}+\left(y_{E}-y_{C}\right)^{2}+\left(z_{E}-z_{C}\right)^{2} \\
l_{5}^{2}=\left(x_{F}-x_{C}\right)^{2}+\left(y_{F}-y_{C}\right)^{2}+\left(z_{F}-z_{C}\right)^{2} \\
l_{6}^{2}=\left(x_{F}-x_{A}\right)^{2}+\left(y_{F}-y_{A}\right)^{2}+\left(z_{F}-z_{A}\right)^{2}
\end{array}\right.
$$

Relations system (79) it is derived by time and one obtains the expressions system (80), from that is explained the linear velocities of the motor elements (81).

$$
\begin{gathered}
\left\{\begin{array}{l}
2 \cdot l_{1} \cdot \dot{l}_{1}=2 \cdot\left(x_{D}-x_{A}\right) \cdot \dot{x}_{D}+2 \cdot\left(y_{D}-y_{A}\right) \cdot \dot{y}_{D}+2 \cdot\left(z_{D}-z_{A}\right) \cdot \dot{z}_{D} \\
2 \cdot l_{2} \cdot \dot{l}_{2}=2 \cdot\left(x_{D}-x_{B}\right) \cdot \dot{x}_{D}+2 \cdot\left(y_{D}-y_{B}\right) \cdot \dot{y}_{D}+2 \cdot\left(z_{D}-z_{B}\right) \cdot \dot{z}_{D} \\
2 \cdot l_{3} \cdot \dot{l}_{3}=2 \cdot\left(x_{E}-x_{B}\right) \cdot \dot{x}_{E}+2 \cdot\left(y_{E}-y_{B}\right) \cdot \dot{y}_{E}+2 \cdot\left(z_{E}-z_{B}\right) \cdot \dot{z}_{E} \\
2 \cdot l_{4} \cdot \dot{l}_{4}=2 \cdot\left(x_{E}-x_{C}\right) \cdot \dot{x}_{E}+2 \cdot\left(y_{E}-y_{C}\right) \cdot \dot{y}_{E}+2 \cdot\left(z_{E}-z_{C}\right) \cdot \dot{z}_{E} \\
2 \cdot l_{5} \cdot \dot{l}_{5}=2 \cdot\left(x_{F}-x_{C}\right) \cdot \dot{x}_{F}+2 \cdot\left(y_{F}-y_{C}\right) \cdot \dot{y}_{F}+2 \cdot\left(z_{F}-z_{C}\right) \cdot \dot{z}_{F} \\
2 \cdot l_{6} \cdot \dot{l}_{6}=2 \cdot\left(x_{F}-x_{A}\right) \cdot \dot{x}_{F}+2 \cdot\left(y_{F}-y_{A}\right) \cdot \dot{y}_{F}+2 \cdot\left(z_{F}-z_{A}\right) \cdot \dot{z}_{F}
\end{array}\right. \\
\left\{\begin{array}{l}
i_{1}=\frac{\left(x_{D}-x_{A}\right) \cdot \dot{x}_{D}+\left(y_{D}-y_{A}\right) \cdot \dot{y}_{D}+\left(z_{D}-z_{A}\right) \cdot \dot{z}_{D}}{l_{1}} \\
i_{2}=\frac{\left(x_{D}-x_{B}\right) \cdot \dot{x}_{D}+\left(y_{D}-y_{B}\right) \cdot \dot{y}_{D}+\left(z_{D}-z_{B}\right) \cdot \dot{z}_{D}}{l_{2}} \\
i_{3}=\frac{\left(x_{E}-x_{B}\right) \cdot \dot{x}_{E}+\left(y_{E}-y_{B}\right) \cdot \dot{y}_{E}+\left(z_{E}-z_{B}\right) \cdot \dot{z}_{E}}{l_{3}} \\
i_{4}=\frac{\left(x_{E}-x_{C}\right) \cdot \dot{x}_{E}+\left(y_{E}-y_{C}\right) \cdot \dot{y}_{E}+\left(z_{E}-z_{C}\right) \cdot \dot{z}_{E}}{l_{4}} \\
i_{5}=\frac{\left(x_{F}-x_{C}\right) \cdot \dot{x}_{F}+\left(y_{F}-y_{C}\right) \cdot \dot{y}_{F}+\left(z_{F}-z_{C}\right) \cdot \dot{z}_{F}}{l_{5}} \\
i_{6}=\frac{\left(x_{F}-x_{A}\right) \cdot \dot{x}_{F}+\left(y_{F}-y_{A}\right) \cdot \dot{y}_{F}+\left(z_{F}-z_{A}\right) \cdot \dot{z}_{F}}{l_{6}}
\end{array}\right.
\end{gathered}
$$

\section{Determining the accelerations}

One knows: $\ddot{x}_{S}, \ddot{y}_{S}, \ddot{z}_{S}, \ddot{\alpha}, \ddot{\beta}, \ddot{\gamma}, \ddot{z}_{D}$.

One starts from the velocities relations (82), aranged in the form (83). 


$$
\begin{aligned}
& \left\{\begin{array}{l}
\left(\dot{x}_{D}-\dot{x}_{S}\right) \cdot \alpha+\left(x_{D}-x_{S}\right) \cdot \dot{\alpha}+\left(\dot{y}_{D}-\dot{y}_{S}\right) \cdot \beta+ \\
+\left(y_{D}-y_{S}\right) \cdot \dot{\beta}=\left(\dot{z}_{S}-\dot{z}_{D}\right) \cdot \gamma+\left(z_{S}-z_{D}\right) \cdot \dot{\gamma} \\
\left(x_{D}-x_{S}\right) \cdot\left(\dot{x}_{D}-\dot{x}_{S}\right)+\left(y_{D}-y_{S}\right) \cdot\left(\dot{y}_{D}-\dot{y}_{S}\right)= \\
=-\left(z_{D}-z_{S}\right) \cdot\left(\dot{z}_{D}-\dot{z}_{S}\right)
\end{array}\right. \\
& \left\{\begin{array}{l}
\alpha \cdot \dot{x}_{D}+\beta \cdot \dot{y}_{D}=\alpha \cdot \dot{x}_{S}-\left(x_{D}-x_{S}\right) \cdot \dot{\alpha}+\beta \cdot \dot{y}_{S}- \\
-\left(y_{D}-y_{S}\right) \cdot \dot{\beta}+\left(\dot{z}_{S}-\dot{z}_{D}\right) \cdot \gamma+\left(z_{S}-z_{D}\right) \cdot \dot{\gamma} \\
\left(x_{D}-x_{S}\right) \cdot \dot{x}_{D}+\left(y_{D}-y_{S}\right) \cdot \dot{y}_{D}=\left(x_{D}-x_{S}\right) \cdot \dot{x}_{S}+ \\
+\left(y_{D}-y_{S}\right) \cdot \dot{y}_{S}-\left(z_{D}-z_{S}\right) \cdot\left(\dot{z}_{D}-\dot{z}_{S}\right)
\end{array}\right.
\end{aligned}
$$

Expressions (83) are derived by time and one obtains the accelerations system (84) which can be arranged to the form (85).

$$
\begin{aligned}
& \left\{\begin{array}{l}
\dot{\alpha} \cdot \dot{x}_{D}+\alpha \cdot \ddot{x}_{D}+\dot{\beta} \cdot \dot{y}_{D}+\beta \cdot \ddot{y}_{D}=\dot{\alpha} \cdot \dot{x}_{S}+\alpha \cdot \ddot{x}_{S}- \\
-\left(\dot{x}_{D}-\dot{x}_{S}\right) \cdot \dot{\alpha}-\left(x_{D}-x_{S}\right) \cdot \ddot{\alpha}+\dot{\beta} \cdot \dot{y}_{S}+\beta \cdot \ddot{y}_{S}- \\
-\left(\dot{y}_{D}-\dot{y}_{S}\right) \cdot \dot{\beta}-\left(y_{D}-y_{S}\right) \cdot \ddot{\beta}+\left(\ddot{z}_{S}-\ddot{z}_{D}\right) \cdot \gamma+ \\
+\left(\dot{z}_{S}-\dot{z}_{D}\right) \cdot \dot{\gamma}+\left(\dot{z}_{S}-\dot{z}_{D}\right) \cdot \dot{\gamma}+\left(z_{S}-z_{D}\right) \cdot \ddot{\gamma} \\
\left(\dot{x}_{D}-\dot{x}_{S}\right) \cdot \dot{x}_{D}+\left(x_{D}-x_{S}\right) \cdot \ddot{x}_{D}+\left(\dot{y}_{D}-\dot{y}_{S}\right) \cdot \dot{y}_{D}+ \\
\left(y_{D}-y_{S}\right) \cdot \ddot{y}_{D}=\left(\dot{x}_{D}-\dot{x}_{S}\right) \cdot \dot{x}_{S}+\left(x_{D}-x_{S}\right) \cdot \ddot{x}_{S}+ \\
+\left(\dot{y}_{D}-\dot{y}_{S}\right) \cdot \dot{y}_{S}+\left(y_{D}-y_{S}\right) \cdot \ddot{y}_{S}- \\
-\left(\dot{z}_{D}-\dot{z}_{S}\right)^{2}-\left(z_{D}-z_{S}\right) \cdot\left(\ddot{z}_{D}-\ddot{z}_{S}\right)
\end{array}\right. \\
& \left\{\begin{array}{l}
\alpha \cdot \ddot{x}_{D}+\beta \cdot \ddot{y}_{D}=2 \cdot \dot{\alpha} \cdot\left(\dot{x}_{S}-\dot{x}_{D}\right)+2 \cdot \dot{\beta} \cdot\left(\dot{y}_{S}-\dot{y}_{D}\right)+ \\
+\alpha \cdot \ddot{x}_{S}+\beta \cdot \ddot{y}_{S}+\left(x_{S}-x_{D}\right) \cdot \ddot{\alpha}+\left(y_{S}-y_{D}\right) \cdot \ddot{\beta}+ \\
+\left(\ddot{z}_{S}-\ddot{z}_{D}\right) \cdot \gamma+2 \cdot\left(\dot{z}_{S}-\dot{z}_{D}\right) \cdot \dot{\gamma}+\left(z_{S}-z_{D}\right) \cdot \ddot{\gamma} \\
\left(x_{D}-x_{S}\right) \cdot \ddot{x}_{D}+\left(y_{D}-y_{S}\right) \cdot \ddot{y}_{D}=-\left(\dot{x}_{D}-\dot{x}_{S}\right)^{2}- \\
-\left(\dot{y}_{D}-\dot{y}_{S}\right)^{2}-\left(\dot{z}_{D}-\dot{z}_{S}\right)^{2}+\left(x_{D}-x_{S}\right) \cdot \ddot{x}_{S}+ \\
+\left(y_{D}-y_{S}\right) \cdot \ddot{y}_{S}-\left(z_{D}-z_{S}\right) \cdot\left(\ddot{z}_{D}-\ddot{z}_{S}\right)
\end{array}\right.
\end{aligned}
$$

One can identify the linear system of two equations with two unknowns (86) with coefficients (87) and solutions (88).

$$
\left\{\begin{array}{l}
a_{11} \cdot \ddot{x}_{D}+a_{12} \cdot \ddot{y}_{D}=f_{1} \\
a_{21} \cdot \ddot{x}_{D}+a_{22} \cdot \ddot{y}_{D}=f_{2}
\end{array}\right.
$$




$$
\left\{\begin{array}{l}
\left\{\begin{array}{l}
a_{11}=\alpha ; \quad a_{12}=\beta ; \quad a_{21}=x_{D}-x_{S} ; \quad a_{22}=y_{D}-y_{S} ; \\
f_{1}=2 \cdot\left[\dot{\alpha} \cdot\left(\dot{x}_{S}-\dot{x}_{D}\right)+\dot{\beta} \cdot\left(\dot{y}_{S}-\dot{y}_{D}\right)+\dot{\gamma} \cdot\left(\dot{z}_{S}-\dot{z}_{D}\right)\right]+\alpha \cdot \ddot{x}_{S}+\beta \cdot \ddot{y}_{S}+ \\
+\gamma \cdot\left(\ddot{z}_{S}-\ddot{z}_{D}\right)+\left(x_{S}-x_{D}\right) \cdot \ddot{\alpha}+\left(y_{S}-y_{D}\right) \cdot \ddot{\beta}+\left(z_{S}-z_{D}\right) \cdot \ddot{\gamma} \\
f_{2}=-\left(\dot{x}_{D}-\dot{x}_{S}\right)^{2}-\left(\dot{y}_{D}-\dot{y}_{S}\right)^{2}-\left(\dot{z}_{D}-\dot{z}_{S}\right)^{2}+ \\
+\left(x_{D}-x_{S}\right) \cdot \ddot{x}_{S}+\left(y_{D}-y_{S}\right) \cdot \ddot{y}_{S}-\left(z_{D}-z_{S}\right) \cdot\left(\ddot{z}_{D}-\ddot{z}_{S}\right) \\
\Delta_{f}=\left|\begin{array}{ll}
a_{11} & a_{12} \\
a_{21} & a_{22}
\end{array}\right|=a_{11} \cdot a_{22}-a_{12} \cdot a_{21} \\
\Delta_{x D 2}=\left|\begin{array}{ll}
f_{1} & a_{12} \\
f_{2} & a_{22}
\end{array}\right|=f_{1} \cdot a_{22}-f_{2} \cdot a_{12} \\
\ddot{x}_{D}=\frac{\Delta_{x D 2} ;}{\Delta_{f}} \quad \ddot{y}_{D}=\frac{\Delta_{y D 2}}{\Delta_{f}} \\
\Delta_{y D 2}=\left|\begin{array}{ll}
a_{11} & f_{1} \\
a_{21} & f_{2}
\end{array}\right|=f_{2} \cdot a_{11}-f_{1} \cdot a_{21}
\end{array}\right.
\end{array}\right.
$$

The velocities system (89) is derived by time and one obtains the accelerations relations (90), which are arranged to the form (91).

$$
\begin{gathered}
\left\{\begin{array}{l}
c_{11} \cdot \dot{x}_{E}+c_{12} \cdot \dot{y}_{E}+c_{13} \cdot \dot{z}_{E}=c_{1} \\
c_{21} \cdot \dot{x}_{E}+c_{22} \cdot \dot{y}_{E}+c_{23} \cdot \dot{z}_{E}=c_{2} \\
c_{31} \cdot \dot{x}_{E}+c_{32} \cdot \dot{y}_{E}+c_{33} \cdot \dot{z}_{E}=c_{3}
\end{array}\right. \\
\left\{\begin{array}{l}
\dot{c}_{11} \cdot \dot{x}_{E}+\dot{c}_{12} \cdot \dot{y}_{E}+\dot{c}_{13} \cdot \dot{z}_{E}+c_{11} \cdot \ddot{x}_{E}+ \\
+c_{12} \cdot \ddot{y}_{E}+c_{13} \cdot \ddot{z}_{E}=\dot{c}_{1} \\
\dot{c}_{21} \cdot \dot{x}_{E}+\dot{c}_{22} \cdot \dot{y}_{E}+\dot{c}_{23} \cdot \dot{z}_{E}+c_{21} \cdot \ddot{x}_{E}+ \\
+c_{22} \cdot \ddot{y}_{E}+c_{23} \cdot \ddot{z}_{E}=\dot{c}_{2} \\
\dot{c}_{31} \cdot \dot{x}_{E}+\dot{c}_{32} \cdot \dot{y}_{E}+\dot{c}_{33} \cdot \dot{z}_{E}+c_{31} \cdot \ddot{x}_{E}+ \\
+c_{32} \cdot \ddot{y}_{E}+c_{33} \cdot \ddot{z}_{E}=\dot{c}_{3}
\end{array}\right.
\end{gathered}
$$




$$
\left\{\begin{array}{l}
c_{11} \cdot \ddot{x}_{E}+c_{12} \cdot \ddot{y}_{E}+c_{13} \cdot \ddot{z}_{E}= \\
=\dot{c}_{1}-\dot{c}_{11} \cdot \dot{x}_{E}-\dot{c}_{12} \cdot \dot{y}_{E}-\dot{c}_{13} \cdot \dot{z}_{E} \\
c_{21} \cdot \ddot{x}_{E}+c_{22} \cdot \ddot{y}_{E}+c_{23} \cdot \ddot{z}_{E}= \\
=\dot{c}_{2}-\dot{c}_{21} \cdot \dot{x}_{E}-\dot{c}_{22} \cdot \dot{y}_{E}-\dot{c}_{23} \cdot \dot{z}_{E} \\
c_{31} \cdot \ddot{x}_{E}+c_{32} \cdot \ddot{y}_{E}+c_{33} \cdot \ddot{z}_{E}= \\
=\dot{c}_{3}-\dot{c}_{31} \cdot \dot{x}_{E}-\dot{c}_{32} \cdot \dot{y}_{E}-\dot{c}_{33} \cdot \dot{z}_{E}
\end{array}\right.
$$

It identifies the coefficients (92) and the linear system (93) consists of three equations of the first degree each, with three unknowns, which is resolved by the relationships (94).

$$
\left\{\begin{array}{l}
c_{11}=\alpha ; \quad \dot{c}_{11}=\dot{\alpha} ; \quad c_{12}=\beta ; \quad \dot{c}_{12}=\dot{\beta} ; \quad c_{13}=\gamma ; \quad \dot{c}_{13}=\dot{\gamma} ; \\
c_{21}=x_{E}-x_{S} ; \quad \dot{c}_{21}=\dot{x}_{E}-\dot{x}_{S} ; \quad c_{22}=y_{E}-y_{S} ; \quad \dot{c}_{22}=\dot{y}_{E}-\dot{y}_{S} ; \\
c_{23}=z_{E}-z_{S} ; \quad \dot{c}_{23}=\dot{z}_{E}-\dot{z}_{S} ; \quad c_{31}=x_{E}-x_{D} ; \quad \dot{c}_{31}=\dot{x}_{E}-\dot{x}_{D} ; \\
c_{32}=y_{E}-y_{D} ; \quad \dot{c}_{32}=\dot{y}_{E}-\dot{y}_{D} ; \quad c_{33}=z_{E}-z_{D} ; \quad \dot{c}_{33}=\dot{z}_{E}-\dot{z}_{D} ; \\
c_{1}=\alpha \cdot \dot{x}_{S}-\left(x_{E}-x_{S}\right) \cdot \dot{\alpha}+\beta \cdot \dot{y}_{S}-\left(y_{E}-y_{S}\right) \cdot \dot{\beta}+\gamma \cdot \dot{z}_{S}-\left(z_{E}-z_{S}\right) \cdot \dot{\gamma} \\
\dot{c}_{1}=\dot{\alpha} \cdot \dot{x}_{S}+\alpha \cdot \ddot{x}_{S}-\left(\dot{x}_{E}-\dot{x}_{S}\right) \cdot \dot{\alpha}-\left(x_{E}-x_{S}\right) \cdot \ddot{\alpha}+\dot{\beta} \cdot \dot{y}_{S}+\beta \cdot \ddot{y}_{S}- \\
-\left(\dot{y}_{E}-\dot{y}_{S}\right) \cdot \dot{\beta}-\left(y_{E}-y_{S}\right) \cdot \ddot{\beta}+\dot{\gamma} \cdot \dot{z}_{S}+\gamma \cdot \ddot{z}_{S}-\left(\dot{z}_{E}-\dot{z}_{S}\right) \cdot \dot{\gamma}-\left(z_{E}-z_{S}\right) \cdot \ddot{\gamma} \\
c_{2}=\left(x_{E}-x_{S}\right) \cdot \dot{x}_{S}+\left(y_{E}-y_{S}\right) \cdot \dot{y}_{S}+\left(z_{E}-z_{S}\right) \cdot \dot{z}_{S} \\
\dot{c}_{2}=\left(\dot{x}_{E}-\dot{x}_{S}\right) \cdot \dot{x}_{S}+\left(x_{E}-x_{S}\right) \cdot \ddot{x}_{S}+\left(\dot{y}_{E}-\dot{y}_{S}\right) \cdot \dot{y}_{S}+\left(y_{E}-y_{S}\right) \cdot \ddot{y}_{S}+ \\
+\left(\dot{z}_{E}-\dot{z}_{S}\right) \cdot \dot{z}_{S}+\left(z_{E}-z_{S}\right) \cdot \ddot{z}_{S} \\
c_{3}=\left(x_{E}-x_{D}\right) \cdot \dot{x}_{D}+\left(y_{E}-y_{D}\right) \cdot \dot{y}_{D}+\left(z_{E}-z_{D}\right) \cdot \dot{z}_{D} \\
\dot{c}_{3}=\left(\dot{x}_{E}-\dot{x}_{D}\right) \cdot \dot{x}_{D}+\left(x_{E}-x_{D}\right) \cdot \ddot{x}_{D}+\left(\dot{y}_{E}-\dot{y}_{D}\right) \cdot \dot{y}_{D}+\left(y_{E}-y_{D}\right) \cdot \ddot{y}_{D}+ \\
+\left(\dot{z}_{E}-\dot{z}_{D}\right) \cdot \dot{z}_{D}+\left(z_{E}-z_{D}\right) \cdot \ddot{z}_{D} \\
e_{1}=\dot{c}_{1}-\dot{c}_{11} \cdot \dot{x}_{E}-\dot{c}_{12} \cdot \dot{y}_{E}-\dot{c}_{13} \cdot \dot{z}_{E} \\
e_{2}=\dot{c}_{2}-\dot{c}_{21} \cdot \dot{x}_{E}-\dot{c}_{22} \cdot \dot{y}_{E}-\dot{c}_{23} \cdot \dot{z}_{E} \\
e_{3}=\dot{c}_{3}-\dot{c}_{31} \cdot \dot{x}_{E}-\dot{c}_{32} \cdot \dot{y}_{E}-\dot{c}_{33} \cdot \dot{z}_{E}
\end{array}\right.
$$




$$
\left\{\begin{array}{l}
c_{11} \cdot \ddot{x}_{E}+c_{12} \cdot \ddot{y}_{E}+c_{13} \cdot \ddot{z}_{E}=e_{1} \\
c_{21} \cdot \ddot{x}_{E}+c_{22} \cdot \ddot{y}_{E}+c_{23} \cdot \ddot{z}_{E}=e_{2} \\
c_{31} \cdot \ddot{x}_{E}+c_{32} \cdot \ddot{y}_{E}+c_{33} \cdot \ddot{z}_{E}=e_{3}
\end{array}\right.
$$

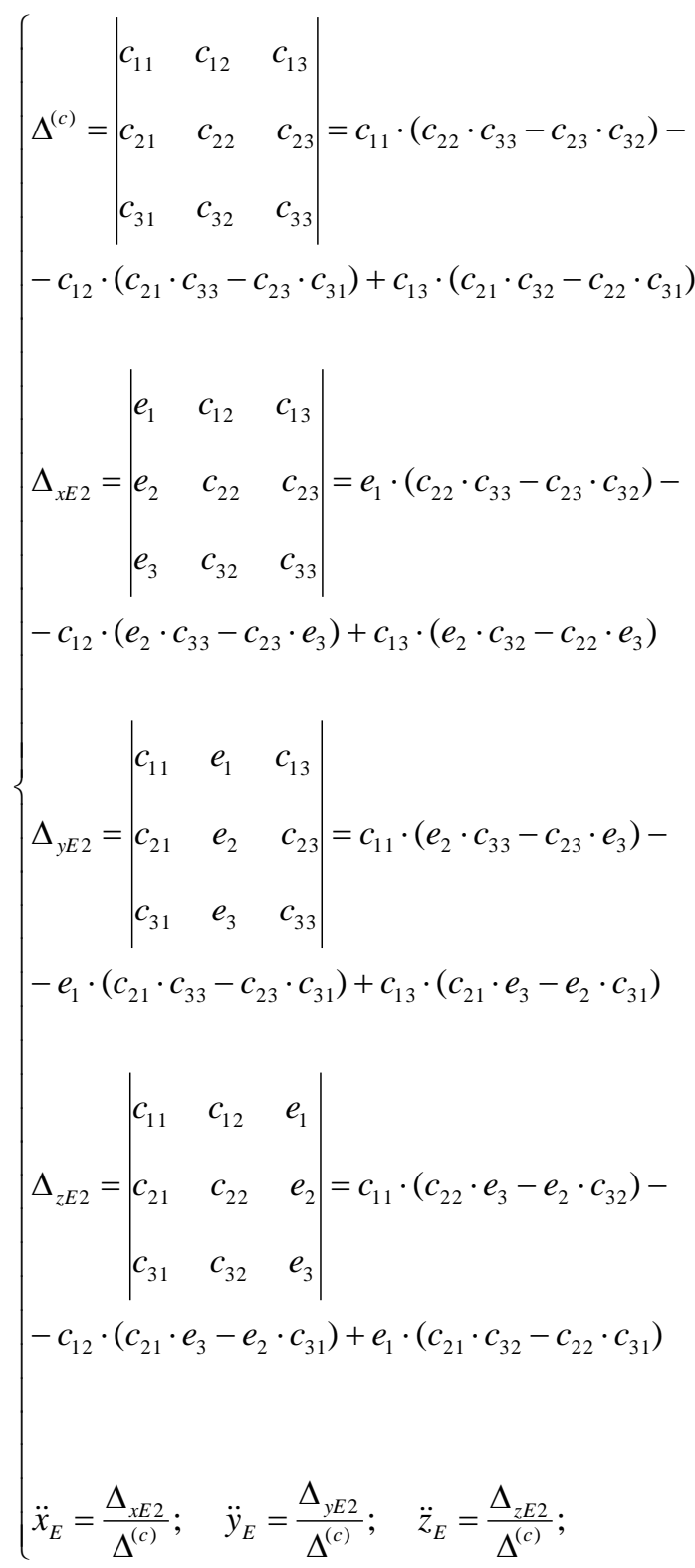

In the following write gear system (95) which is derived by time to obtains the accelerations system (96) which is arranged in the form (97). 


$$
\begin{gathered}
\left\{\begin{array}{l}
d_{11} \cdot \dot{x}_{F}+d_{12} \cdot \dot{y}_{F}+d_{13} \cdot \dot{z}_{F}=d_{1} \\
d_{21} \cdot \dot{x}_{F}+d_{22} \cdot \dot{y}_{F}+d_{23} \cdot \dot{z}_{F}=d_{2} \\
d_{31} \cdot \dot{x}_{F}+d_{32} \cdot \dot{y}_{F}+d_{33} \cdot \dot{z}_{F}=d_{3}
\end{array}\right. \\
\left\{\begin{array}{l}
\dot{d}_{11} \cdot \dot{x}_{F}+\dot{d}_{12} \cdot \dot{y}_{F}+\dot{d}_{13} \cdot \dot{z}_{F}+d_{11} \cdot \ddot{x}_{F}+ \\
+d_{12} \cdot \ddot{y}_{F}+d_{13} \cdot \ddot{z}_{F}=\dot{d}_{1} \\
\dot{d}_{21} \cdot \dot{x}_{F}+\dot{d}_{22} \cdot \dot{y}_{F}+\dot{d}_{23} \cdot \dot{z}_{F}+d_{21} \cdot \ddot{x}_{F}+ \\
+d_{22} \cdot \ddot{y}_{F}+d_{23} \cdot \ddot{z}_{F}=\dot{d}_{2} \\
\dot{d}_{31} \cdot \dot{x}_{F}+\dot{d}_{32} \cdot \dot{y}_{F}+\dot{d}_{33} \cdot \dot{z}_{F}+d_{31} \cdot \ddot{x}_{F}+ \\
+d_{32} \cdot \ddot{y}_{F}+d_{33} \cdot \ddot{z}_{F}=\dot{d}_{3} \\
\left\{\begin{array}{l}
d_{11} \cdot \ddot{x}_{F}+d_{12} \cdot \ddot{y}_{F}+d_{13} \cdot \ddot{z}_{F}= \\
\dot{d}_{1}-\dot{d}_{11} \cdot \dot{x}_{F}-\dot{d}_{12} \cdot \dot{y}_{F}-\dot{d}_{13} \cdot \dot{z}_{F} \\
d_{21} \cdot \ddot{x}_{F}+d_{22} \cdot \ddot{y}_{F}+d_{23} \cdot \ddot{z}_{F}= \\
=\dot{d}_{2}-\dot{d}_{21} \cdot \dot{x}_{F}-\dot{d}_{22} \cdot \dot{y}_{F}-\dot{d}_{23} \cdot \dot{z}_{F} \\
=\dot{x}_{3}-\dot{d}_{31} \cdot \dot{x}_{F}-\dot{d}_{32} \cdot \dot{y}_{F}-\dot{d}_{33} \cdot \dot{z}_{F}
\end{array}\right.
\end{array}\right. \\
\dot{y}_{F}+d_{33} \cdot \ddot{z}_{F}=
\end{gathered}
$$

The coefficients are determined by relations (98) and the system takes the form (99). 


$$
\left\{\begin{array}{l}
d_{11}=\alpha ; \quad \dot{d}_{11}=\dot{\alpha} ; \quad d_{12}=\beta ; \quad \dot{d}_{12}=\dot{\beta} ; \quad d_{13}=\gamma ; \quad \dot{d}_{13}=\dot{\gamma} ; \\
d_{1}=\alpha \cdot \dot{x}_{S}+\beta \cdot \dot{y}_{S}+\gamma \cdot \dot{z}_{S}-\left(x_{F}-x_{S}\right) \cdot \dot{\alpha}-\left(y_{F}-y_{S}\right) \cdot \dot{\beta}-\left(z_{F}-z_{S}\right) \cdot \dot{\gamma} ; \\
\dot{d}_{1}=\dot{\alpha} \cdot \dot{x}_{S}+\alpha \cdot \ddot{x}_{S}+\dot{\beta} \cdot \dot{y}_{S}+\beta \cdot \ddot{y}_{S}+\dot{\gamma} \cdot \dot{z}_{S}+\gamma \cdot \ddot{z}_{S}-\left(\dot{x}_{F}-\dot{x}_{S}\right) \cdot \dot{\alpha}- \\
-\left(x_{F}-x_{S}\right) \cdot \ddot{\alpha}-\left(\dot{y}_{F}-\dot{y}_{S}\right) \cdot \dot{\beta}-\left(y_{F}-y_{S}\right) \cdot \ddot{\beta}-\left(\dot{z}_{F}-\dot{z}_{S}\right) \cdot \dot{\gamma}-\left(z_{F}-z_{S}\right) \cdot \ddot{\gamma} ; \\
d_{21}=x_{F}-x_{S} ; \quad d_{22}=y_{F}-y_{S} ; \quad d_{23}=z_{F}-z_{S} ; \\
\dot{d}_{21}=\dot{x}_{F}-\dot{x}_{S} ; \quad \dot{d}_{22}=\dot{y}_{F}-\dot{y}_{S} ; \quad \dot{d}_{23}=\dot{z}_{F}-\dot{z}_{S} ; \\
d_{2}=\left(x_{F}-x_{S}\right) \cdot \dot{x}_{S}+\left(y_{F}-y_{S}\right) \cdot \dot{y}_{S}+\left(z_{F}-z_{S}\right) \cdot \dot{z}_{S} ; \\
\dot{d}_{2}=\left(\dot{x}_{F}-\dot{x}_{S}\right) \cdot \dot{x}_{S}+\left(x_{F}-x_{S}\right) \cdot \ddot{x}_{S}+\left(\dot{y}_{F}-\dot{y}_{S}\right) \cdot \dot{y}_{S}+ \\
+\left(y_{F}-y_{S}\right) \cdot \ddot{y}_{S}+\left(\dot{z}_{F}-\dot{z}_{S}\right) \cdot \dot{z}_{S}+\left(z_{F}-z_{S}\right) \cdot \ddot{z}_{S} ; \\
d_{31}=x_{F}-x_{D} ; \quad d_{32}=y_{F}-y_{D} ; \quad d_{33}=z_{F}-z_{D} ; \\
\dot{d}_{31}=\dot{x}_{F}-\dot{x}_{D} ; \quad \dot{d}_{32}=\dot{y}_{F}-\dot{y}_{D} ; \quad \dot{d}_{33}=\dot{z}_{F}-\dot{z}_{D} ; \\
d_{3}=\left(x_{F}-x_{D}\right) \cdot \dot{x}_{D}+\left(y_{F}-y_{D}\right) \cdot \dot{y}_{D}+\left(z_{F}-z_{D}\right) \cdot \dot{z}_{D} ; \\
\dot{d}_{3}=\left(\dot{x}_{F}-\dot{x}_{D}\right) \cdot \dot{x}_{D}+\left(x_{F}-x_{D}\right) \cdot \ddot{x}_{D}+\left(\dot{y}_{F}-\dot{y}_{D}\right) \cdot \dot{y}_{D}+ \\
+\left(y_{F}-y_{D}\right) \cdot \ddot{y}_{D}+\left(\dot{z}_{F}-\dot{z}_{D}\right) \cdot \dot{z}_{D}+\left(z_{F}-z_{D}\right) \cdot \ddot{z}_{D} ; \\
g_{1}=\dot{d}_{1}-\dot{d}_{11} \cdot \dot{x}_{F}-\dot{d}_{12} \cdot \dot{y}_{F}-\dot{d}_{13} \cdot \dot{z}_{F} ; \\
g_{2}=\dot{d}_{2}-\dot{d}_{21} \cdot \dot{x}_{F}-\dot{d}_{22} \cdot \dot{y}_{F}-\dot{d}_{23} \cdot \dot{z}_{F} ; \\
g_{3}=\dot{d}_{3}-\dot{d}_{31} \cdot \dot{x}_{F}-\dot{d}_{32} \cdot \dot{y}_{F}-\dot{d}_{33} \cdot \dot{z}_{F}
\end{array}\right.
$$

The system (99) with coefficients (98) is solved with the relations (100). 


$$
\begin{aligned}
& \left\{\begin{array}{l}
\Delta^{(g)}=\left|\begin{array}{ccc}
d_{11} & d_{12} & d_{13} \\
d_{21} & d_{22} & d_{23} \\
d_{31} & d_{32} & d_{33}
\end{array}\right|=d_{11} \cdot\left(d_{22} \cdot d_{33}-d_{23} \cdot d_{32}\right)- \\
-d_{12} \cdot\left(d_{21} \cdot d_{33}-d_{23} \cdot d_{31}\right)+d_{13} \cdot\left(d_{21} \cdot d_{32}-d_{22} \cdot d_{31}\right)
\end{array}\right. \\
& \Delta_{x F 2}=\left|\begin{array}{ccc}
g_{1} & d_{12} & d_{13} \\
g_{2} & d_{22} & d_{23} \\
g_{3} & d_{32} & d_{33}
\end{array}\right|=g_{1} \cdot\left(d_{22} \cdot d_{33}-d_{23} \cdot d_{32}\right)- \\
& -d_{12} \cdot\left(g_{2} \cdot d_{33}-d_{23} \cdot g_{3}\right)+d_{13} \cdot\left(g_{2} \cdot d_{32}-d_{22} \cdot g_{3}\right) \\
& \Delta_{y F 2}=\left|\begin{array}{lll}
d_{11} & g_{1} & d_{13} \\
d_{21} & g_{2} & d_{23} \\
d_{31} & g_{3} & d_{33}
\end{array}\right|=d_{11} \cdot\left(g_{2} \cdot d_{33}-d_{23} \cdot g_{3}\right)- \\
& -g_{1} \cdot\left(d_{21} \cdot d_{33}-d_{23} \cdot d_{31}\right)+d_{13} \cdot\left(d_{21} \cdot g_{3}-g_{2} \cdot d_{31}\right) \\
& \begin{array}{l}
\Delta_{z F 2}=\left|\begin{array}{ccc}
d_{11} & d_{12} & g_{1} \\
d_{21} & d_{22} & g_{2} \\
d_{31} & d_{32} & g_{3}
\end{array}\right|=d_{11} \cdot\left(d_{22} \cdot g_{3}-\right. \\
-d_{12} \cdot\left(d_{21} \cdot g_{3}-g_{2} \cdot d_{31}\right)+g_{1} \cdot\left(d_{21} \cdot d_{32}\right. \\
\ddot{x}_{F}=\frac{\Delta_{x F 2}}{\Delta^{(g)}} ; \quad \ddot{y}_{F}=\frac{\Delta_{y F 2}}{\Delta^{(g)}} ; \quad \ddot{z}_{F}=\frac{\Delta_{z F 2}}{\Delta^{(g)}} ;
\end{array}
\end{aligned}
$$

It writes now the linear velocities system (102) obtained by deriving position system (101). The system (102) derived generates in turn the linear accelerations system (103). 
ISSN: $1415-7314$

ISSN online: 2317-6717

$$
\begin{aligned}
& \left\{\begin{array}{l}
l_{1}^{2}=\left(x_{D}-x_{A}\right)^{2}+\left(y_{D}-y_{A}\right)^{2}+\left(z_{D}-z_{A}\right)^{2} \\
l_{2}^{2}=\left(x_{D}-x_{B}\right)^{2}+\left(y_{D}-y_{B}\right)^{2}+\left(z_{D}-z_{B}\right)^{2} \\
l_{3}^{2}=\left(x_{E}-x_{B}\right)^{2}+\left(y_{E}-y_{B}\right)^{2}+\left(z_{E}-z_{B}\right)^{2} \\
l_{4}^{2}=\left(x_{E}-x_{C}\right)^{2}+\left(y_{E}-y_{C}\right)^{2}+\left(z_{E}-z_{C}\right)^{2} \\
l_{5}^{2}=\left(x_{F}-x_{C}\right)^{2}+\left(y_{F}-y_{C}\right)^{2}+\left(z_{F}-z_{C}\right)^{2} \\
l_{6}^{2}=\left(x_{F}-x_{A}\right)^{2}+\left(y_{F}-y_{A}\right)^{2}+\left(z_{F}-z_{A}\right)^{2}
\end{array}\right. \\
& \left\{\begin{array}{l}
l_{1} \cdot \dot{l}_{1}=\left(x_{D}-x_{A}\right) \cdot \dot{x}_{D}+\left(y_{D}-y_{A}\right) \cdot \dot{y}_{D}+\left(z_{D}-z_{A}\right) \cdot \dot{z}_{D} \\
l_{2} \cdot \dot{l}_{2}=\left(x_{D}-x_{B}\right) \cdot \dot{x}_{D}+\left(y_{D}-y_{B}\right) \cdot \dot{y}_{D}+\left(z_{D}-z_{B}\right) \cdot \dot{z}_{D} \\
l_{3} \cdot \dot{l}_{3}=\left(x_{E}-x_{B}\right) \cdot \dot{x}_{E}+\left(y_{E}-y_{B}\right) \cdot \dot{y}_{E}+\left(z_{E}-z_{B}\right) \cdot \dot{z}_{E} \\
l_{4} \cdot \dot{l}_{4}=\left(x_{E}-x_{C}\right) \cdot \dot{x}_{E}+\left(y_{E}-y_{C}\right) \cdot \dot{y}_{E}+\left(z_{E}-z_{C}\right) \cdot \dot{z}_{E} \\
l_{5} \cdot \dot{l}_{5}=\left(x_{F}-x_{C}\right) \cdot \dot{x}_{F}+\left(y_{F}-y_{C}\right) \cdot \dot{y}_{F}+\left(z_{F}-z_{C}\right) \cdot \dot{z}_{F} \\
l_{6} \cdot \dot{l}_{6}=\left(x_{F}-x_{A}\right) \cdot \dot{x}_{F}+\left(y_{F}-y_{A}\right) \cdot \dot{y}_{F}+\left(z_{F}-z_{A}\right) \cdot \dot{z}_{F}
\end{array}\right.
\end{aligned}
$$




$$
\left\{\begin{array}{l}
\dot{l}_{1}^{2}+l_{1} \cdot \ddot{l}_{1}=\left(\dot{x}_{D}-\dot{x}_{A}\right) \cdot \dot{x}_{D}+\left(x_{D}-x_{A}\right) \cdot \ddot{x}_{D}+\left(\dot{y}_{D}-\dot{y}_{A}\right) \cdot \dot{y}_{D}+ \\
+\left(y_{D}-y_{A}\right) \cdot \ddot{y}_{D}+\left(\dot{z}_{D}-\dot{z}_{A}\right) \cdot \dot{z}_{D}+\left(z_{D}-z_{A}\right) \cdot \ddot{z}_{D} \\
i_{2}^{2}+l_{2} \cdot \ddot{l}_{2}=\left(\dot{x}_{D}-\dot{x}_{B}\right) \cdot \dot{x}_{D}+\left(x_{D}-x_{B}\right) \cdot \ddot{x}_{D}+\left(\dot{y}_{D}-\dot{y}_{B}\right) \cdot \dot{y}_{D}+ \\
+\left(y_{D}-y_{B}\right) \cdot \ddot{y}_{D}+\left(\dot{z}_{D}-\dot{z}_{B}\right) \cdot \dot{z}_{D}+\left(z_{D}-z_{B}\right) \cdot \ddot{z}_{D} \\
i_{3}^{2}+l_{3} \cdot \ddot{l}_{3}=\left(\dot{x}_{E}-\dot{x}_{B}\right) \cdot \dot{x}_{E}+\left(x_{E}-x_{B}\right) \cdot \ddot{x}_{E}+\left(\dot{y}_{E}-\dot{y}_{B}\right) \cdot \dot{y}_{E}+ \\
+\left(y_{E}-y_{B}\right) \cdot \ddot{y}_{E}+\left(\dot{z}_{E}-\dot{z}_{B}\right) \cdot \dot{z}_{E}+\left(z_{E}-z_{B}\right) \cdot \ddot{z}_{E} \\
i_{4}^{2}+l_{4} \cdot \ddot{l}_{4}=\left(\dot{x}_{E}-\dot{x}_{C}\right) \cdot \dot{x}_{E}+\left(x_{E}-x_{C}\right) \cdot \ddot{x}_{E}+\left(\dot{y}_{E}-\dot{y}_{C}\right) \cdot \dot{y}_{E}+ \\
+\left(y_{E}-y_{C}\right) \cdot \ddot{y}_{E}+\left(\dot{z}_{E}-\dot{z}_{C}\right) \cdot \dot{z}_{E}+\left(z_{E}-z_{C}\right) \cdot \ddot{z}_{E} \\
i_{5}^{2}+l_{5} \cdot \ddot{l}_{5}=\left(\dot{x}_{F}-\dot{x}_{C}\right) \cdot \dot{x}_{F}+\left(x_{F}-x_{C}\right) \cdot \ddot{x}_{F}+\left(\dot{y}_{F}-\dot{y}_{C}\right) \cdot \dot{y}_{F}+ \\
+\left(y_{F}-y_{C}\right) \cdot \ddot{y}_{F}+\left(\dot{z}_{F}-\dot{z}_{C}\right) \cdot \dot{z}_{F}+\left(z_{F}-z_{C}\right) \cdot \ddot{z}_{F} \\
i_{6}^{2}+l_{6} \cdot \ddot{l}_{6}=\left(\dot{x}_{F}-\dot{x}_{A}\right) \cdot \dot{x}_{F}+\left(x_{F}-x_{A}\right) \cdot \ddot{x}_{F}+\left(\dot{y}_{F}-\dot{y}_{A}\right) \cdot \dot{y}_{F}+ \\
+\left(y_{F}-y_{A}\right) \cdot \ddot{y}_{F}+\left(\dot{z}_{F}-\dot{z}_{A}\right) \cdot \dot{z}_{F}+\left(z_{F}-z_{A}\right) \cdot \ddot{z}_{F}
\end{array}\right.
$$

From the system (103) is explicit linear accelerations (104) corresponding to the six feet mobile that supports and acts the superior mobile platform DEF. 


$$
\left\{\begin{array}{l}
\ddot{l}_{1}=\left[\left(\dot{x}_{D}-\dot{x}_{A}\right) \cdot \dot{x}_{D}+\left(x_{D}-x_{A}\right) \cdot \ddot{x}_{D}+\left(\dot{y}_{D}-\dot{y}_{A}\right) \cdot \dot{y}_{D}+\right. \\
\left.+\left(y_{D}-y_{A}\right) \cdot \ddot{y}_{D}+\left(\dot{z}_{D}-\dot{z}_{A}\right) \cdot \dot{z}_{D}+\left(z_{D}-z_{A}\right) \cdot \ddot{z}_{D}-\dot{l}_{1}^{2}\right] / l_{1} \\
\ddot{l}_{2}=\left[\left(\dot{x}_{D}-\dot{x}_{B}\right) \cdot \dot{x}_{D}+\left(x_{D}-x_{B}\right) \cdot \ddot{x}_{D}+\left(\dot{y}_{D}-\dot{y}_{B}\right) \cdot \dot{y}_{D}+\right. \\
\left.+\left(y_{D}-y_{B}\right) \cdot \ddot{y}_{D}+\left(\dot{z}_{D}-\dot{z}_{B}\right) \cdot \dot{z}_{D}+\left(z_{D}-z_{B}\right) \cdot \ddot{z}_{D}-\dot{l}_{2}^{2}\right] / l_{2} \\
\ddot{l}_{3}=\left[\left(\dot{x}_{E}-\dot{x}_{B}\right) \cdot \dot{x}_{E}+\left(x_{E}-x_{B}\right) \cdot \ddot{x}_{E}+\left(\dot{y}_{E}-\dot{y}_{B}\right) \cdot \dot{y}_{E}+\right. \\
\left.+\left(y_{E}-y_{B}\right) \cdot \ddot{y}_{E}+\left(\dot{z}_{E}-\dot{z}_{B}\right) \cdot \dot{z}_{E}+\left(z_{E}-z_{B}\right) \cdot \ddot{z}_{E}-\dot{l}_{3}^{2}\right] / l_{3} \\
\ddot{l}_{4}=\left[\left(\dot{x}_{E}-\dot{x}_{C}\right) \cdot \dot{x}_{E}+\left(x_{E}-x_{C}\right) \cdot \ddot{x}_{E}+\left(\dot{y}_{E}-\dot{y}_{C}\right) \cdot \dot{y}_{E}+\right. \\
\left.+\left(y_{E}-y_{C}\right) \cdot \ddot{y}_{E}+\left(\dot{z}_{E}-\dot{z}_{C}\right) \cdot \dot{z}_{E}+\left(z_{E}-z_{C}\right) \cdot \ddot{z}_{E}-\dot{l}_{4}^{2}\right] / l_{4} \\
\ddot{l}_{5}=\left[\left(\dot{x}_{F}-\dot{x}_{C}\right) \cdot \dot{x}_{F}+\left(x_{F}-x_{C}\right) \cdot \ddot{x}_{F}+\left(\dot{y}_{F}-\dot{y}_{C}\right) \cdot \dot{y}_{F}+\right. \\
\left.+\left(y_{F}-y_{C}\right) \cdot \ddot{y}_{F}+\left(\dot{z}_{F}-\dot{z}_{C}\right) \cdot \dot{z}_{F}+\left(z_{F}-z_{C}\right) \cdot \ddot{z}_{F}-\dot{l}_{5}^{2}\right] / l_{5} \\
\ddot{l}_{6}=\left[\left(\dot{x}_{F}-\dot{x}_{A}\right) \cdot \dot{x}_{F}+\left(x_{F}-x_{A}\right) \cdot \ddot{x}_{F}+\left(\dot{y}_{F}-\dot{y}_{A}\right) \cdot \dot{y}_{F}+\right. \\
\left.+\left(y_{F}-y_{A}\right) \cdot \ddot{y}_{F}+\left(\dot{z}_{F}-\dot{z}_{A}\right) \cdot \dot{z}_{F}+\left(z_{F}-z_{A}\right) \cdot \ddot{z}_{F}-\dot{l}_{6}^{2}\right] / l_{6}
\end{array}\right.
$$

\section{Applications}

Presented system can be useful in particular to the surgical robots that operate patients who require an accuracy of positioning very high (see figure 13).

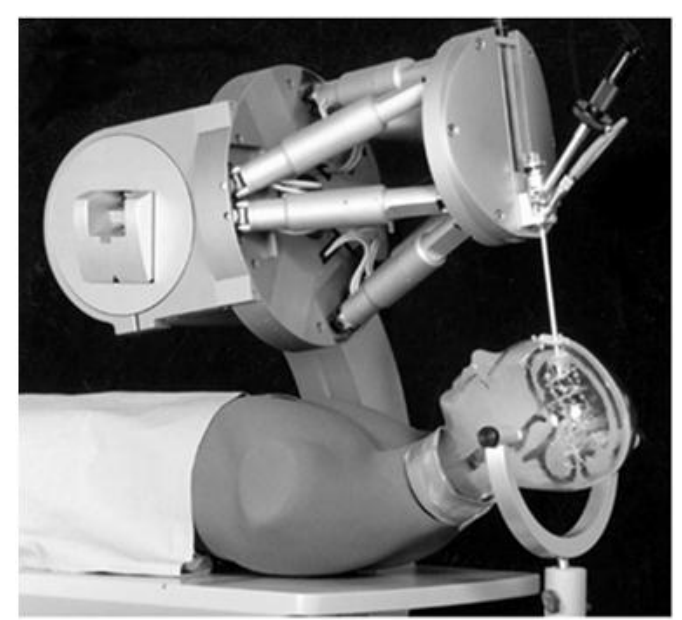

Fig. 13 A surgical robot Stewart system

These platforms can position very accurately even very large weights, such as a telescope modern stationary (see Fig. 14). 


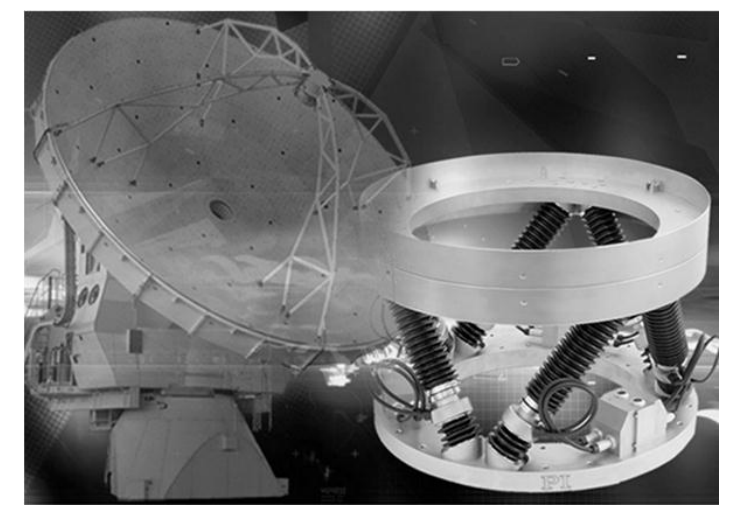

Fig. 14 A modern stationary telescope positioned by a Stewart system

Other applications of the platform Stewart are handling and precise positioning of objects large and heavy.

Spatial Stewart platforms may conquer outer space in the future (MELO; ALVES; ROSÁRIO, 2012).

The latest PC-based digital controllers, facilitated by open-interface architecture providing a variety of high-level commands, allow choosing any point in space as the pivot point for the rotation axes by software command (TANG; SUN; SHAO, 2013). Target positions in 6space are specified in Cartesian coordinates, and the controller transforms them into the required motion-vectors for the individual actuator drives. Any position and any orientation can be entered directly, and the specified target will be reached by a smooth vector motion. The pivot point then remains fixed relative to the platform (TABAKOVIĆ et al., 2013).

\section{Discussion}

In addition to the coordinated output of the six hexapod axes, these new hexapod controllers provide two additional axes that can be used to operate rotary stages, linear stages or linear actuators. Some include a macro language for programming and storing command sequences. These controllers feature flexible interfaces, such as TCP/IP interface for remote, network and Internet connection.

New simulation tools are being incorporated for graphical configuration and simulation of hexapods to verify workspace requirements and loads. Such software provides full functionality for creation, modeling, simulation, rendering and playback of hexapod configurations to predict and avoid interference with possible obstacles in the workspace.

With the new design developments that hexapod systems are experiencing, manufacturers and researchers that have a need for extreme high resolutions and high accuracy can now capitalize on them for improvements within their workplace. Hexapod technology has advanced considerably in a few short years, now it is up to industry to embrace these new developments and put them to work to reduce their set-up and processing time, overall production cycle times, and ultimately reduced cost of operation. 


\section{Conclusions}

The presented method manages to synthesize (in theory) the best option parameters for any desired parallel system. Moving mechanical systems parallel structures are solid, fast, and accurate. Between parallel systems (WANG et al., 2013) it is to be noticed Stewart platforms, as the oldest systems, fast, solid and precise.

The work outlines a few main elements of Stewart platforms. Begin with the geometry platform, structure, and continue with kinematic elements of it (inverse kinematic) with positions, velocities and accelerations.

If a structural motto element consists of two moving elements which translates relative, drive train and especially dynamic it is more convenient to represent the motto element as a single moving components. We have thus seven moving parts (the six motto elements or feet to which is added mobile platform 7) and one fixed.

Proposed method (in this work) may determine kinematic parameters system position when required the co-ordinates of the endeffector $\mathrm{S}$.

This is clearly a reverse motion (an inverse kinematics) (LIN et al., 2013).

\section{References}

CAO, W.; DING, H.; BIN, ZI; ZIMING, CHEN (2013). New Structural Representation and Digital-Analysis Platform for Symmetrical Parallel Mechanisms, International Journal of Advanced Robotic Systems, Sumeet S Aphale (Ed.), ISBN: 1729-8806, InTech, DOI: 10.5772/56380. Available from:

http://www.intechopen.com/journals/international_journal_of_advanced_robotic_systems/newstructural-representation-and-digital-analysis-platform-for-symmetrical-parallel-mechanisms.

MELO, L. F.; ALVES, S. F. R.; ROSÁRIO, J. M. (2012) Mobile Robot Navigation Modelling, Control and Applications, in International Review on Modelling and Simulations, April 2012, v. 5, n. 2B, p. 1059-1068. Available from:

http://www.praiseworthyprize.com/IREMOS-latest/IREMOS_vol_5_n_2.html.

LEE, B. J. (2013). Geometrical Derivation of Differential Kinematics to Calibrate Model Parameters of Flexible Manipulator, International Journal of Advanced Robotic Systems, Jaime Gallardo-Alvarado, Ramon Rodriguez-Castro (Ed.), ISBN: 1729-8806, InTech, DOI: 10.5772/55592. Available from:

http://www.intechopen.com/journals/international_journal_of_advanced_robotic_systems/geom etrical-derivation-of-differential-kinematics-to-calibrate-model-parameters-of-flexible-

manipula.

LIN, W.; BING LI; XIAOJUN Y.; DAN Z. (2013). Modelling and Control of Inverse Dynamics for a 5-DOF Parallel Kinematic Polishing Machine, International Journal of Advanced Robotic Systems, Sumeet S Aphale (Ed.), ISBN: 1729-8806, InTech, DOI: 10.5772/54966. Available from:

http://www.intechopen.com/journals/international_journal_of_advanced_robotic_systems/mode lling-and-control-of-inverse-dynamics-for-a-5-dof-parallel-kinematic-polishing-machine. 
GARCIA, E.; JIMENEZ, M. A.; SANTOS, P. G.; ARMADA, M. (2007) The evolution of robotics research, Robotics \& Automation Magazine, IEEE , v.14, n.1, p.90-103, March 2007. Available from:

http://ieeexplore.ieee.org/stamp/stamp.jsp?tp=\&arnumber=4141037\&isnumber=4141014.

GARCIA, M. M.; GALLARDO, A. J.; CASTILLO C. E. (2013). Finding the Generalized Forces of a Series-Parallel Manipulator, IJARS, Jaime Gallardo-Alvarado, Ramon Rodriguez-Castro (Ed.), ISBN: 1729-8806, InTech, DOI: 10.5772/53824. Available from:

http://www.intechopen.com/journals/international_journal_of_advanced_robotic_systems/findin g-the-generalized-forces-of-a-series-parallel-manipulator.

HE, B.; WANG, Z.; LI, Q.; XIE, H.; SHEN, R. (2013). An Analytic Method for the Kinematics and Dynamics of a Multiple-Backbone Continuum Robot, IJARS, Patricia Melin (Ed.), ISBN: 1729-8806, InTech, DOI: 10.5772/54051. Available from:

http://www.intechopen.com/journals/international_journal_of_advanced_robotic_systems/ananalytic-method-for-the-kinematics-and-dynamics-of-a-multiple-backbone-continuum-robot.

LIU, H.; ZHOU, W.; LAI, X.; ZHU, S. (2013). An Efficient Inverse Kinematic Algorithm for a PUMA560-Structured Robot Manipulator, IJARS, Jaime Gallardo-Alvarado, Ramon RodriguezCastro (Ed.), ISBN: 1729-8806, InTech, DOI: 10.5772/56403. Available from:

http://www.intechopen.com/journals/international_journal_of_advanced_robotic_systems/anefficient-inverse-kinematic-algorithm-for-a-puma560-structured-robot-manipulator.

PADULA, F.; PERDEREAU, V., (2013). An On-Line Path Planner for Industrial Manipulators, International Journal of Advanced Robotic Systems, Antonio Visioli (Ed.), ISBN: 1729-

8806, InTech, DOI: 10.5772/55063. Available from:

http://www.intechopen.com/journals/international_journal_of_advanced_robotic_systems/anon-line-path-planner-for-industrial-manipulators.

PERUMAAL, S.; JAWAHAR, N., (2013). Automated Trajectory Planner of Industrial Robot for Pick-and-Place Task, IJARS, Antonio Visioli (Ed.), ISBN: 1729-8806, InTech, DOI: 10.5772/53940. Available from:

http://www.intechopen.com/journals/international_journal_of_advanced_robotic_systems/auto mated-trajectory-planner-of-industrial-robot-for-pick-and-place-task.

PETRESCU, F. I., PETRESCU, R. V. (2013) Cinematics of the 3R Dyad, in journal Engevista, v. 15, n. 2, p. 118-124, August 2013, ISSN 1415-7314. Available from:

http://www.uff.br/engevista/seer/index.php/engevista/article/view/376.

PETRESCU, F. I., PETRESCU, R. V. (2012) Kinematics of the Planar Quadrilateral Mechanism, in journal Engevista, v. 14, n. 3, p. 345-348, December 2012, ISSN 1415-7314. Available from: http://www.uff.br/engevista/seer/index.php/engevista/article/view/377.

PETRESCU, F. I., PETRESCU, R. V. (2012) Mecatronica - Sisteme Seriale si Paralele, Create Space publisher, USA, March 2012, ISBN 978-1-4750-6613-5, 128 pages, Romanian edition.

PETRESCU, F. I, PETRESCU, R. V (2011) Mechanical Systems, Serial and Parallel - Course (in romanian), LULU Publisher, London, UK, February 2011, 124 pages, ISBN 978-1-44660039-9, Romanian edition.

PETRESCU, F. I., GRECU, B., COMANESCU, A., PETRESCU, R. V. (2009) Some Mechanical Design Elements. In the 3rd International Conference on Computational Mechanics and Virtual Engineering, COMEC 2009, Braşov, October 2009, ISBN 978-973-598-572-1, Edit. UTB, p. 520-525.

REDDY, P.; SHIHABUDHEEN K. V.; JACOB, J. (2012) Precise Non Linear Modeling of Flexible Link Flexible Joint Manipulator, in International Review on Modelling and Simulations, June 2012, v. 5, n. 3B, p. 1368-1374. Available from:

http://www.praiseworthyprize.com/IREMOS-latest/IREMOS_vol_5_n_3.html.

TABAKOVIĆ, S.; MILAN, Z.; RATKO, G.; ŽIVKOVIĆ, A. (2013). 
Program Suite for Conceptual Designing of Parallel Mechanism-Based Robots and Machine Tools, International Journal of Advanced Robotic Systems, Sumeet S Aphale (Ed.), ISBN: 1729-8806, InTech, DOI: 10.5772/56633. Available from:

http://www.intechopen.com/journals/international_journal_of_advanced_robotic_systems/progr am-suite-for-conceptual-designing-of-parallel-mechanism-based-robots-and-machine-tools.

TANG, X.; SUN, D.; SHAO, Z., (2013). The Structure and Dimensional Design of a Reconfigurable PKM, IJARS, Sumeet S Aphale (Ed.), ISBN: 1729-8806, InTech, DOI: 10.5772/54696. Available from:

http://www.intechopen.com/journals/international_journal_of_advanced_robotic_systems/thestructure-and-dimensional-design-of-a-reconfigurable-pkm.

TONG, G.; GU, J.; XIE, W. (2013). Virtual Entity-Based Rapid Prototype for Design and Simulation of Humanoid Robots, International Journal of Advanced Robotic Systems, Guangming Xie (Ed.), ISBN: 1729-8806, InTech, DOI: 10.5772/55936. Available from:

http://www.intechopen.com/journals/international_journal_of_advanced_robotic_systems/virtua l-entity-based-rapid-prototype-for-design-and-simulation-of-humanoid-robots.

WANG, K.; LUO, M.; MEI, T.; ZHAO, J.; CAO, Y., (2013). Dynamics Analysis of a Three-DOF Planar Serial-Parallel Mechanism for Active Dynamic Balancing with Respect to a Given Trajectory, International Journal of Advanced Robotic Systems, Sumeet S Aphale (Ed.), ISBN: 1729-8806, InTech, DOI: 10.5772/54201. Available from:

http://www.intechopen.com/journals/international_journal_of_advanced_robotic_systems/dyna mics-analysis-of-a-three-dof-planar-serial-parallel-mechanism-for-active-dynamic-balancingwith-. 